\title{
Dynamics and Functional Interplay of Non-histone Lysine Crotonylome and Ubiquitylome in Vascular Smooth Muscle Cell Phenotypic Remodeling
}

\section{Shan-Hu Cao}

Hebei Medical University

\section{Zhi-Huan Chen}

Hebei Medical University

Lin Yue

Hebei Medical University

Han-Mei Jiang

Hebei Medical University

Ru-Yuan Ma

Hebei Medical University

Li-Hua Dong ( $\square$ langchao1979@126.com )

Hebei Medical University https://orcid.org/0000-0002-4039-963X

\section{Research}

Keywords: vascular smooth muscle cells, phenotypic remodeling, lysine crotonylation, lysine ubiquitylation, PPI analysis

Posted Date: September 14th, 2021

DOl: https://doi.org/10.21203/rs.3.rs-864586/v1

License: (c) (i) This work is licensed under a Creative Commons Attribution 4.0 International License. Read Full License 


\section{Abstract}

\section{Backgrounds}

Crotonylation of histones is a recently discovered type of post-translational modification that can regulate gene expression. However, the function of crotonylation on nonhistone proteins in vascular smooth muscle cells (VSMC) is unclear. Here, we aim to use modification and proteomic analysis to find the cellular characteristic of crotonylated nonhistone proteins and the crosstalk with ubiquitinated proteins in vascular smooth muscle cell (VSMC) phenotypic remodeling.

Results

We performed modification and proteomic analysis of VSMCs before and after platelet-derived growth factor-BB (PDGF-BB) stimulation. The crotonylated and ubiquitinated pan-antibody was used to enrich the protein and then subjected to high-throughput mass spectrometry analysis. Then we compared the enrichment analysis of differentially modified proteins in regards to GO terms, KEGG pathway and protein domain. As a result, there were 2138 crotonylation sites in 534 proteins and 1359 ubiquitination sites corresponding to 657 proteins. The crotonylated proteins participate in a variety of important cellular pathways and perform different functions in VSMCs. Among them, some proteins were found to be closely involved in the physiological process of VSMC phenotypic remodeling including glycolysis/gluconeogenesis, vascular smooth muscle contraction, and PI3K-Akt signaling pathway. Furthermore, the KEGG pathway enrichment analysis showed that the ubiquitinated proteins were found to be closely involved in the physiological process of VSMC phenotypic remodeling including glycolysis/gluconeogenesis, vascular smooth muscle contraction, RAS signaling pathway or PI3K-Akt signaling pathway. Crosstalk analysis showed that there were 199 sites within 177 proteins modified by crotonylation and ubiquitination simultaneously. PPI network analysis indicated that crotonylated and ubiquitinated proteins played an important role in cellular bioprocess commonly and possible synergistic effect.

\section{Conclusions}

In summary, our bioinformatics show that nonhistone crotonylation and ubiquitination play an important role in the VSMC phenotypic transformation induced by PDGF-BB stimulation. The crosstalk of crotonylation and ubiquitination in glycolysis is possibly a novel mechanism underlying the VSMC phenotypic remodeling.

\section{Background}

Cardiovascular diseases are the primary cause of death among patients all over the world, and the risk factors of cardiovascular disease are gradually increasing among young people [1]. Vascular smooth muscle cells (VSMCs) carry out phenotypic switching when blood vessels are injured, and phenotypic transformation is the first step of the pathological basis of vascular diseases [2]. Phenotypic 
transformation of smooth muscle cells leads to vascular remodeling when vascular diseases occur, such as atherosclerosis, diabetic macroangiopathy, and restenosis [3,4].

After vascular injury, the serum with growth factors, cytokines and other components contacting smooth muscle cells activate complex signaling pathways and cellular events. Among the events of cellular activation, numerous studies have focused on growth factors which could contribute to the formation of atherosclerotic and restenosis lesions $[5,6]$. We focus our study exclusively on the role of platelet-derived growth factor-BB (PDGF-BB) whose function is as chemoattractant or mitogen for VSMC $[7,8]$. When the blood vessel is enduring injury, PDGF-BB could lead to phenotypic modulation and the cellular gene expression involved in pattern-changing is even more [9].

Post-translational modifications (PTMs) are the most foundational and complicated mechanisms that could regulate various cellular events, such as gene expression, protein synthesis, metabolism, and cell cycle [10-12]. he extensive interplay between kinase pathways that regulate the VSMC phenotypic remodeling is heavily regulated by several PTMs. Our research group provides evidence that Tumor necrosis factor receptor-associated factor 6 (TRAF6)-induced smooth muscle 22a (SM22a) ubiquitination maintains VSMC survival through increased G6PD activity and GAPDH production [13]. Recently, we found that TNF-a concurrently caused SIRT1 phosphorylation by virtue of CKII and protected VSMC from inflammation [14]. Phosphorylation of SIRT1 make it to interact with and deacetylate EZH2 and, thereafter, promote SM22a transcription via EZH2 activity suppression [14]. PTMs mainly occur at the lysine residue on account of its specific spatial structure. Besides several well-studied types of lysine acetylation [15], malonylation [16], ubiquitination [17], and methylation [18], lysine crotonylation (Kcr) is a newly identified and approved type of PTM, where a crotonyl group is transferred to a lateral chain of lysine.

Lysine crotonylation is one of PTMs of histone proteins originally identified by Tan et al. This novel evolutionarily conserved histone modification was identified on 28 lysine sites on various histones [19]. Similar with histone acetylation, crotonylation also occurs on the $\varepsilon$-amino group of lysine but distinguishes itself from acetylation by its four-carbon length and planar orientation. Histone crotonylation specifically labels the enhancers and transcription start sites of active genes in both the human somatic cell genome and the murine male germ cell genome. Histone crotonylation affects chromatin structure and facilitates histone replacement in elongating spermatids [19]. Lysine crotonylation, but not acetylation, preferentially marks "escape genes" during post-meiotic sex inactivation in mouse testes [20]. Lysine crotonylation and acetylation sites overlap in histones and are catalyzed through p300/CBP, a well-known histone acetyltransferase [21]. Then, several regulatory enzymes for histone crotonylation have been described, such as histone acetyltransferase (HAT) p300 [21], HDAC3 [22] and SIRT1/2/3 [23-26].

Recently, the advancements in the mass spectrometry technology and crotonyl-peptides enrichment methods facilitated the identification of hundreds to thousands of crotonylation sites in both prokaryotes and eukaryotes. The proteome analysis of lysine crotonylation has been reported in HEK293 cell [27] and 
mouse liver [28] and maintenance hemodialysis patients [29]. This vital aspect has substantially extended our understanding of protein crotonylation. However, there are still relatively few studies on the crotonylation of non-histone proteins. Recently, Xu et al. describe heretofore unidentified crotonylation of non-histone proteins. They showed that some enzymes that catalyze acetylation or deacetylation also mediate crotonylation or decrotonylation of non-histone proteins. These non-histone proteins are involved in diverse cellular functions and signaling pathways. Identification of crotonylation on non-histone proteins expands our understanding of this PTM at a proteomics scale and may facilitate the elucidation of the precise modulation of protein functions [30].

Ubiquitylation is an another PTM, in which the small protein ubiquitin is covalently attached to lysine of substrate proteins. This modification can mark substrates for degradation by the proteasome or lysosomes, or alternatively can alter protein localization, function, trafficking, and protein-protein interactions. For example, tumor necrosis factor receptor-associated factor 6 (TRAF6), a downstream effector of interleukin-1 $\beta$ (IL-1 $\beta$ ), triggers YAP ubiquitination at K252, which interrupts the interaction between YAP and angiomotin and results in enhanced YAP nuclear translocation [31]. The ubiquitinmediated signaling is frequently altered in cancer [32]. Chu et al. provides evidence that an E3 ubiquitin ligase complex, linear ubiquitin chain assembly complex (LUBAC) and a deubiquitinating enzyme (DUB) OTULIN cooperatively regulate autophagy initiation and autophagosome maturation by mediating the linear ubiquitination and the stabilization of ATG13 [33]. However, little is known about the extent and role of ubiquitylation during VSMC phenotypic remodeling, and possible protein lysine co-modification by various moieties has not been explored in cardiovascular system.

In this work, we established an integrated system by the combination of SILAC labeling, affinity enrichment by antibodies, and high-resolution liquid chromatography-tandem MS (LC-MS/MS) for a quantitative comparison of the crotonylation and ubiquitylation of VSMCs before and after PDGF-BB treatment. So far, there is no relevant research on crotonylation and ubiqutination and their crosstalk analysis in cardiovascular diseases. This whole proteome and modified-omics comparison can provide a starting point for understanding the pathogenesis of vascular diseases associated to VSMC phenotypic remodeling.

\section{Results}

\section{Quantitative comparison of the crotonylome of VSMCs before and after PDGF-BB treatment}

It is demonstrated that PDGF-BB could regulate VSMC differentiation at the concentration of 10 $\mathrm{ng} / \mathrm{ml}$ [34]. Then, VSMCs were incubated with PDGF-BB (10 ng/ml) for 1, 3, 6, 12, 24, 48 and $72 \mathrm{~h}$. We examined the changes of several post-translational modifications during the phenotypic transformation of VSMC. As shown in Figure S1a, the expression of the smooth muscle contractile proteins SM a-actin and SM22a decreased at $12 \mathrm{~h}$, which indicated VSMC transformation toward a proliferative phenotype at $12 \mathrm{~h}$ after PDGF-BB-stimulating. Moreover, the pan-crotonylated level increased at $3 \mathrm{~h}$ after PDGF-BBstimulated and peaked at $24 \mathrm{~h}$ (Figure S1b). For other types of post-translational modifications, with the 
prolonged time of PDGF-BB stimulation, there is no consistency in the level of post-translational modification and the phenotypic transformation (Figure S1c-h). Subsequently, the proteins derived from VSMCs before and after PDGF-BB treatment were separately identified using liquid chromatography and mass spectrometry (LC-MS/MS)-based quantitative analysis within three random repetitions according to the experimental flowchart (Figure 1a).

For biological replicates or technical replicates, we test whether the quantitative results of biological replicates or technical replicates are statistically consistent. The heat map shows an obvious positive correlation between sample replicates and a negative correlation between different treatment groups (Figure 1b). Another quantitation heat map shows that there is a high similarity between duplicate samples but modification sites have changed significantly after PDGF-BB treatment (Figure 1c). To validate the MS data, we then checked the mass error of the identified peptides. The distribution of mass errors was near zero, and most errors were $<0.02 \mathrm{Da}$, indicating the accuracy of the MS data (Figure 1d).

In this work, 2386 crotonylation sites corresponding to 570 proteins were identified and 2138 crotonylation sites from 534 proteins were quantified by the comparison between VSMCs with and without PDGF-BB treatment (Table 1). Among the 534 quantifiable proteins, 245 crotonylation sites from 125 proteins changed over 1.3 folds (200 sites from 94 proteins up-regulated and 45 sites from 31 proteins down-regulated) (Figure 1e). We also performed statistics on the proteins and sites corresponding to other fold differences (Table 2). Of these 570 crotonylated proteins, $233(40.9 \%)$ have only one crotonylation site, 94 (16.5\%) contain two sites, and $67(11.8 \%)$ possess three. $58(10.2 \%)$ proteins get 10 more crotonylation sites (Figure 1f), such as pyruvate kinase, which includes 16 crotonylated sites (Table 3 ).

Table 1. MS/MS spectrum database search analysis summary of crotonylomics

\begin{tabular}{|llll|}
\hline $\begin{array}{l}\text { Identified } \\
\text { proteins }\end{array}$ & Quantifiable proteins & $\begin{array}{l}\text { Identified } \\
\text { sites }\end{array}$ & $\begin{array}{l}\text { Quantifiable } \\
\text { sites }\end{array}$ \\
\hline 570 & 534 & 2368 & 2138 \\
\hline
\end{tabular}

Table 2. Differentially changed modification sites (modified proteins) summary of crotonylomics (filtered with threshold value of expression fold change)

\begin{tabular}{|c|c|c|c|c|c|}
\hline \multirow[t]{2}{*}{$\begin{array}{l}\text { Compare } \\
\text { group }\end{array}$} & $\begin{array}{l}\text { Regulated } \\
\text { type }\end{array}$ & $\begin{array}{l}\text { fold } \\
\text { change }\end{array}$ & $\begin{array}{l}\text { fold } \\
\text { change }\end{array}$ & $\begin{array}{l}\text { fold } \\
\text { change }\end{array}$ & $\begin{array}{l}\text { fold } \\
\text { change }\end{array}$ \\
\hline & & $>1.2$ & $>1.3$ & $>1.5$ & $>2$ \\
\hline \multirow[t]{2}{*}{ PDGF/Con } & up-regulated & 241 (107) & $200(94)$ & $100(58)$ & $14(9)$ \\
\hline & down-regulated & $49(35)$ & $45(31)$ & $31(20)$ & $14(7)$ \\
\hline
\end{tabular}


Table 3. Modification site information of pyruvate kinase

\begin{tabular}{|c|c|c|c|c|c|c|}
\hline Position & $\begin{array}{l}\text { Amino } \\
\text { acid }\end{array}$ & $\begin{array}{l}\text { Protein } \\
\text { description }\end{array}$ & Score & Modified sequence & $\begin{array}{l}\text { Mass } \\
\text { error } \\
\text { [ppm] }\end{array}$ & $\begin{array}{l}\text { MS/MS } \\
\text { Count }\end{array}$ \\
\hline 269 & K & $\begin{array}{l}\text { Pyruvate } \\
\text { kinase }\end{array}$ & 104.05 & VFLAQK(1)MMIGR & 1.4519 & 12 \\
\hline 380 & K & $\begin{array}{l}\text { Pyruvate } \\
\text { kinase }\end{array}$ & 48.686 & VEASFK(1)CCSGAIIVLTK & 0.84548 & 1 \\
\hline 260 & K & $\begin{array}{l}\text { Pyruvate } \\
\text { kinase }\end{array}$ & 81.525 & K(0.006)VLGEK(0.994)VFLAQK & -2.6483 & 2 \\
\hline 266 & K & $\begin{array}{l}\text { Pyruvate } \\
\text { kinase }\end{array}$ & 104.05 & VFLAQK(1)MMIGR & 1.4519 & 12 \\
\hline 261 & K & $\begin{array}{l}\text { Pyruvate } \\
\text { kinase }\end{array}$ & 91.906 & VLGEK(1)GK & -0.41152 & 5 \\
\hline 305 & K & $\begin{array}{l}\text { Pyruvate } \\
\text { kinase }\end{array}$ & 116.58 & GDLGIEIPAEK(1)VFLAQK & 0.27956 & 23 \\
\hline 115 & K & $\begin{array}{l}\text { Pyruvate } \\
\text { kinase }\end{array}$ & 151.91 & PVAVALDTK(1)GPEIR & -1.5825 & 15 \\
\hline 367 & K & $\begin{array}{l}\text { Pyruvate } \\
\text { kinase }\end{array}$ & 112.26 & IMLSGETAK(1)GDYPLEAVR & 0.53367 & 8 \\
\hline 498 & K & $\begin{array}{l}\text { Pyruvate } \\
\text { kinase }\end{array}$ & 109.15 & VNLAMNVGK(1)AR & 2.312 & 11 \\
\hline 186 & K & $\begin{array}{l}\text { Pyruvate } \\
\text { kinase }\end{array}$ & 97.823 & IYVDDGLISLQVK(1)EK & 2.1065 & 10 \\
\hline 89 & K & $\begin{array}{l}\text { Pyruvate } \\
\text { kinase }\end{array}$ & 124.84 & THEYHAETIK(1)NVR & 1.6963 & 14 \\
\hline 125 & K & $\begin{array}{l}\text { Pyruvate } \\
\text { kinase }\end{array}$ & 67.846 & TGLIK(1)GSGTAEVELK & 0.46409 & 1 \\
\hline 224 & K & $\begin{array}{l}\text { Pyruvate } \\
\text { kinase }\end{array}$ & 137.72 & LPAVSEK(1)DIQDLK & 0.39533 & 19 \\
\hline 62 & K & $\begin{array}{l}\text { Pyruvate } \\
\text { kinase }\end{array}$ & 94.692 & SVEMLK(1)EMIK & 0.82137 & 30 \\
\hline 311 & K & $\begin{array}{l}\text { Pyruvate } \\
\text { kinase }\end{array}$ & 104.05 & VFLAQK(1)MMIGR & 1.4519 & 12 \\
\hline 270 & K & $\begin{array}{l}\text { Pyruvate } \\
\text { kinase }\end{array}$ & 143.97 & IISK(1)IENHEGVR & 0.5539 & 9 \\
\hline
\end{tabular}


To determine whether there are any specific amino acid biases adjacent to crotonylation sites, we analyzed the flanking amino acids of these sites, from -10 to +10 positions of lysine. This analysis showed that there were a total of 2731 peptides containing crotonylation sites. Further characterization of the motifs surrounding lysine crotonylation sites showed a series of distinct patterns of crotonylation motifs (Table 4). Among them, the peptides included 10 specific and conserved motifs, such as .KcrL.......... (368 peptides), ..........KcrE.......... (334 peptides), ..........EKcr.......... (331 peptides) and so on. $\mathrm{Kcr}$ is the meaning of crotonylated lysine, $\mathrm{L}$ leucine, $\mathrm{E}$ glutamic acid, respectively. ".........." represents any single amino acid (Figure S2). Motif enrichment heat map showed glutamic acid (E) and aspartic acid (D) residues were over-enriched at the -1 and +1 positions besides the lysine crotonylation sites (Figure $1 \mathrm{~g}$ ). The distribution of other residues is as follows: alanine $(A)$ is at $-1 /+4$ positions, phenylalanine $(F)$ is at $-1 /+1$, isoleucine $(I)$ and leucine $(L)$ are at $-3 /-4$, arginine $(R)$ is at +2 , and tyrosine $(Y)$ is at -1 . The crotonylation of lysine neutralizes its positive charge and weakens its electrostatic interaction with surrounding negatively charged amino acids, such as $E$ and $D$. These data show for the first time that numerous non-histone proteins are modified by crotonylation in VSMCs.

Table 4. Characteristic sequence of modified site obtained by motif analysis

\begin{tabular}{|c|c|c|c|c|c|c|c|}
\hline \multirow[t]{2}{*}{ Motif } & \multirow[t]{2}{*}{ Motif Score } & \multicolumn{2}{|c|}{ Foreground } & \multicolumn{2}{|c|}{ Background } & \multirow[t]{2}{*}{ Fold Increase } & \multirow{4}{*}{$\begin{array}{l}\text { Enrichment } \\
\text { Analyses of } \\
\text { Lysine }\end{array}$} \\
\hline & & Matches & Size & Matches & Size & & \\
\hline 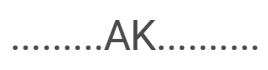 & 16 & 247 & 2270 & 35327 & 601417 & 1.85 & \\
\hline$\ldots \ldots \ldots . . . . . . . . . . .$. & 16 & 149 & 2023 & 17268 & 566090 & 2.41 & \\
\hline$\ldots \ldots \ldots \ldots$ & 14.75 & 277 & 1874 & 49987 & 548822 & 1.62 & \\
\hline$\ldots \ldots \ldots \ldots \ldots \ldots$ & 16 & 190 & 1597 & 31385 & 498835 & 1.89 & \\
\hline$\ldots \ldots \ldots . . . . K E \ldots \ldots . .$. & 12.92 & 238 & 1407 & 48851 & 467450 & 1.62 & \\
\hline 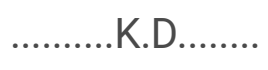 & 9.94 & 100 & 1169 & 17939 & 418599 & 2 & \\
\hline ..........К ............. & 9.62 & 121 & 1069 & 24850 & 400660 & 1.82 & \\
\hline 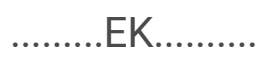 & 6.66 & 137 & 948 & 34965 & 375810 & 1.55 & \\
\hline 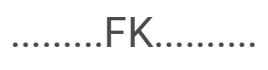 & 6.62 & 60 & 811 & 12313 & 340845 & 2.05 & \\
\hline ……..... YK & 6.49 & 52 & 751 & 10534 & 328532 & 2.16 & \\
\hline
\end{tabular}

\section{Crotonylated Proteins}

To further evaluate crotonylated proteins, we classified these proteins according to their subcellular location, cellular component, molecular function, and associated biological processes. In the up-regulated crotonylated proteins, $46 \%$ were in the cytoplasm, $21 \%$ were in the nucleus, and $13 \%$ were in the mitochondria (Figure S3a). On the contrary, in the down-regulated crotonylated proteins, $32 \%$ were in the cytoplasm, $26 \%$ were in the nucleus, and $13 \%$ were in the mitochondria (Figure S3a). The cellular 
component of crotonylated proteins was classified according to the gene ontology (GO) annotation (Figure S3b). The up-regulated crotonylated proteins were distributed in the cell (22\%), the organelles $(22 \%)$ and the macromolecular complexes $(14 \%)$. However, the down-regulated crotonylated proteins were distributed in the cell (23\%), the organelles $(23 \%)$ and membranes $(16 \%)$. The molecular function of these crotonylated proteins were assigned to several groups based on the $\mathrm{GO}$ annotation. In the up-regulated crotonylated proteins, $53 \%$ were involved in binding, $19 \%$ were related to structural molecule activity, and $18 \%$ were associated with catalytic activity. In the down-regulated crotonylated proteins, $60 \%$ were involved in binding, $15 \%$ were associated with catalytic activity, and $9 \%$ were related to structural molecule activity. Biological process analysis of crotonylated proteins showed that $18 \%$ of up-regulated crotonylated proteins were involved in cellular processes, $13 \%$ were in the single-organism process and $13 \%$ were in the metabolic process. On the contrary, $17 \%$ of down-regulated crotonylated proteins were involved in cellular processes, $16 \%$ were in the single-organism process and $13 \%$ were in biological regulation. These results indicate the relatively broad distributions of crotonylated proteins in subcellular location, cellular component, molecular function, and biological processes.

To investigate the roles of the crotonylation pathways, enrichment analysis was used to classify all the crotonylated protein groups based on the GO databases. GO enrichment analysis of the molecular function showed that up-regulated crotonylated proteins were significantly enriched in the actin-binding, cell adhesion, molecule binding, and structural constituent of ribosome (Figure 2a). The down-regulated crotonylated proteins were significantly enriched in calcium ion binding, metal ion binding, and cation binding (Figure 2b). GO-based functional enrichment analysis of cellular components showed that upregulated crotonylated proteins were significantly enriched in the structural constituents of ribosomes, cytoskeleton, and anchoring junction (Figure 2a). The down-regulated crotonylated proteins were significantly enriched in the extracellular vesicle, extracellular exosome, and extracellular organelle (Figure 2b). Enrichment analysis of biological processes indicated these up-regulated modified proteins were enriched in a variety of biological processes including protein folding, actin cytoskeleton organization, and actin filament-based process (Figure 2a). These down-regulated modified proteins were enriched in a variety of biological processes including protein heterooligomerization, heterotetramerization, and oligomerization (Figure 2b). GO annotation analyses suggest that crotonylated proteins participate in a variety of important cellular pathways and perform different functions in VSMCs.

KEGG enrichment analysis showed that the up-regulated crotonylated proteins were related to multiple metabolic pathways, including ribosome, HTLV-1 infection, biosynthesis of amino acids, and glycolysis/gluconeogenesis (Figure 2c). The pathways involved in down-regulated proteins include multiple metabolic pathways, including arginine and proline metabolism, endoplasmic reticulum, endocytosis, bacterial invasion of epithelial cells, and viral carcinogenesis (Figure 2d). Among them, some proteins were found to be closely involved in the physiological process of VSMC phenotypic remodeling including glycolysis/gluconeogenesis, vascular smooth muscle contraction, and PI3K-Akt signaling pathway. 
Differential protein domain enrichment analysis results showed that many up-regulated proteins had the same amino acid sequence as the calponin homology $(\mathrm{CH})$ domain (Figure 2e). $\mathrm{CH}$ domain is the most common shared amino acid sequence of various actin-binding proteins, which is composed of 6 alphahelices. The $\mathrm{CH}$ domain plays a stabilizing role in cytoskeletal dynamics and can activate downstream pathways in signal transduction [35]. In the present study, there are 6 proteins (Transgelin, Calponin, Microtubule-associated protein RP/EB family member 1, Plectin, Filamin A, IQ motif containing GTPase activating protein 1) containing $\mathrm{CH}$ domain were extensively crotonylated at multiple sites.

According to the KEGG pathway enrichment analysis, the abundances of 21 ribosomal proteins (12 large subunit ribosomal proteins and 9 small subunit ribosomal proteins) involved in the ribosome pathway were differentially expressed in the with and without PDGF-BB-stimulated VSMCs (Figure 2f/S4), which indicated their possible involvement in PDGF-BB induced VSMC phenotypic remodeling. For the enrichment of protein complex, we found one statistically significant SDH-mABC1-PIC-ANT-ATPase complex (Figure $2 \mathrm{~g}$ ), which was in the mitochondrial inner membrane and transported $\mathrm{K}^{+}$with similar characteristics of mitoK $\mathrm{K}_{\text {ATP. }}$. This implies that differentially expressed proteins may be involved in cell mitochondrial activity and thus affect phenotypic transformation.

\section{Cluster analysis of functions of proteins with different fold changes}

To find the correlation of the functions of proteins with different differential expression fold changes, we divide them into 4 parts according to their differential expression multiples, including Q1 to Q4: Q1 (0< Ratio $\leq 1 / 1.5)$, Q2 (1/1.5 < Ratio $\leq 1 / 1.3)$, Q3 (1.3 < Ratio $\leq 1.5)$ and Q4 (Ratio >1.5) (Ratio = PDGF/Con) (Figure S5a). The statistical results show that after PDGF-BB treatment, the number of modification sites in the up-regulated proteins increased significantly.

We then performed enrichment and cluster analysis of GO, KEGG, and protein domains for each Q group. GO enrichment for the Q3 group showed up-regulated proteins were involved in many biological processes, such as response to hypoxia, regulation of protein metabolic process, regulation of endocytosis, positive regulation of cell proliferation, and so on (Figure S5b). And these up-regulated proteins are mainly distributed on the cell surface, cytoskeleton, cytoplasm, and ribosomes (Figure S5c). The molecular function analysis of the up-regulated proteins showed they can perform corresponding functions by combining with cell adhesion factors, RNA, cadherin, ADP (Figure S5d). These results suggest that the fold change maybe one of the factors that affect the function of modification.

\section{STRING interaction network of crotonylated proteins with Cytoscape}

The STRING database was used to search for protein-protein interaction among some crotonylated proteins associated with special complexes. The crotonylated proteins that were functionally clustered and identified belonged to multiple protein complexes, including the ribosome, the $\mathrm{C}$ complex spliceosome, and protein transport. The interaction network was visualized by Cytoscape (Figure 3). 
In this work, ubiquitylome profile of VSMCs before and after PDGF-BB treatment was investigated and three biological replicates were analyzed for each treatment (Figure S6a). Totally, 1532 ubiquitination sites corresponding to 718 proteins were identified, and 1359 ubiquitination sites corresponding to 657 proteins were quantifiable (Table 5). According to a fold change of more than 1.3 or less than $1 / 1.3,124$ sites from 84 proteins were up-regulated and 85 sites from 56 proteins were down-regulated (Table 6). To check our MS data, the quality control was performed, and our results indicated that our MS data satisfied the subsequent advanced analysis (Figure S6b).

Table 5. Identification and quantitative statistics of modification of ubiquitinomics

\begin{tabular}{lll} 
& Identification & Quantitative statistics \\
\hline Sites & 1532 & 1359 \\
\hline Proteins & 718 & 657
\end{tabular}

Table 6. Statistical information of differentially expressed modification level of ubiquitinomics

\begin{tabular}{llll} 
Comparable group & Type & Up $(>1.3)$ & Down $(<1 / 1.3)$ \\
\hline PDGF-vs-Con & Sites & 124 & 85 \\
\cline { 2 - 4 } & Proteins & 84 & 56
\end{tabular}

Subcellular localization analysis showed that the differentially ubiquitination modified proteins were mainly distributed in plasma membrane, cytoplasm and nucleus, and a small amount were distributed in mitochondria, peroxisomes, and cytoskeleton (Figure 4a). To determine the position-specific frequencies of the amino acid residues adjacent to ubiquitination sites, we analyzed the flanking sequences of these sites. We identified two conserved motifs which were designated EK..........., and K........... (Figure 4b). Among them, EK. has been reported in rice [36] and K.......... has been reported to be novel motif in petunia [37]. In the previous study, researchers have doubted that conserved motifs might not exist in mammals because they failed to identify a conserved definition of the ubiquitination site motifs in human [38]. Our work is the first time that reported conserved motifs of the ubiquitination sites in rats. Further analysis of the amino acids neighboring the ubiquitination sites indicated that hydrophilic residues glutamate $(E)$ was overrepresented at the -1 and +3 positions surrounding the lysine ubiquitination sites (Figure 4c).

To further evaluate ubiquitinated proteins that we observed to be up-regulated or down-regulated, these proteins were classified according to their cellular component, molecular function and associated biological processes (Figure 4d/e). GO enrichment analysis of the molecular function showed that upregulated ubiquitinated proteins were significantly enriched in the macromolecular complex binding. The down-regulated ubiquitinated proteins were significantly enriched in cytoskeleton protein binding and nucleotide binding. For the cellular component analysis, up-regulated ubiquitinated proteins were significantly enriched in membrane such as extrinsic component of plasma membrane, endosome membrane, whole membrane and filopodium, suggesting that ubiquitination may play important roles in 
cell membrane. On the contrary, the down-regulated ubiquitinated proteins were significantly enriched in cytoskeleton and microtubule. In the biological process analysis, up-regulated modified proteins were enriched in a variety of biological processes including protein transport, organic substance transport, protein localization, developmental cell growth and cell morphogenesis. These down-regulated modified proteins were enriched in a variety of biological processes including cell morphogenesis, cytoskeleton organization and cell differentiation.

The KEGG pathway enrichment analysis showed that some proteins were found to be closely involved in the physiological process of VSMC phenotypic remodeling including glycolysis/gluconeogenesis, vascular smooth muscle contraction, RAS signaling pathway or PI3K-Akt signaling pathway (Figure 4f). Moreover, ubiquitination of SM22a which can promote VSMC survival was reported in previous study [39]. All these results suggested that protein ubiquitination may possess important roles in phenotypic remodeling of VSMCs.

\section{Enrichment analysis of crotonylated and ubiquitinated proteins.}

To clarify the relationship between crotonylation and ubiquitination, the crosstalk analysis was made on enrichment analysis and other aspects. Statistical analysis showed that before and after PDGF-BB stimulation of VSMC, there were 199 sites within 177 proteins modified by crotonylation and ubiquitination simultaneously (Figure 5a). Then we compared the enrichment analysis of differentially modified proteins in regards to GO terms, KEGG pathway and protein domain. Protein domain analysis revealed that the significant crotonylated one was calponin homology domain which was in proteins that could bind actin and affect cytoskeleton [40] and VHS (Vps-27, Hrs and STAM) domain in ubiquitination which was associated with mono-ubiquitin or poly-ubiquitin chains' covalent binding with cargo receptors [41] and vesicular tracking after membrane recognition [42]. In GO terms and KEGG pathways, we found obvious differences and similarities when comparing the two modifications. The crotonylated proteins were extensively involved in actin cytoskeleton organization and the ubiquitinated proteins are mainly involved in cell morphogenesis (Figure 5b). The cellular component of proteins of crotonylation and ubiquitination differed in cytoskeleton obviously (Figure 5c), in the meantime, the molecular function of crotonylated and ubiquitinated proteins were common in structural molecule activity, cadherin binding and other related binding functions (Figure $5 \mathrm{~d}$ ). And the KEGG pathway analysis indicated that crotonylation and ubiquitination co-affect MAPK signaling pathway, pyruvate metabolism, vascular smooth muscle contraction, Glycolysis/Gluconeogenesis and so on (Figure $5 \mathrm{e}$ ). These results suggest that crotonylation and ubiquitination are jointly involved in certain biological functions of cells.

\section{Crosstalk of PPI network of crotonylated and ubiquitinated proteins.}

We built the protein-protein interaction network of of crotonylated and ubiquitinated proteins, which are significantly differentially expressed, based on the STRING data-base and clustered the proteins on the strength of MCODE (Figure 6/S7). In these clusters, we found some important function clustering including glycolysis/gluconeogenesis, ribosome, vascular smooth muscle contraction, tight junction, regulation of actin cytoskeleton and proteasome. The function cluster was consistent with KEGG 
pathway analysis. In these function clustering, proteins which can be both crotonylated and ubiquitinated will be focused on and could be candidates for future studies, such as Pkm, Ldha, Rps17, Rps27a, Rps12, Rps23, Myh9 and so on. This analysis indicates that crotonylated and ubiquitinated proteins played an important role in cellular bioprocess commonly and possible synergistic effect.

\section{Crotonylation and ubiquitination of enzymes in glucose metabolism.}

According to the KEGG pathway analysis and function clustering based on MCODE, glycolysis/gluconeogenesis are both highlighted (Figure 7). And most enzymes involved in glycolysis are significant ones, because they are both crotonylated and ubiquitinated. Among them, one of the key enzymes, hexokinase, has two crotonylation-modified sites and one ubiquitination-modified site. And another key enzyme, pyruvate kinase, has fourteen crotonylation-modified sites and four ubiquitinationmodified sites. Other important enzymes involved in glycolysis also have varying degrees of modifications. These results suggest that glycolysis possibly change obviously when affected by crotonylation and ubiquitination.

\section{Discussion}

Histone crotonylation, a type of lysine modification, is newly found in mammalian cells, and this modification is significantly enriched in gene promoters and enhancers [19]. Previous studies have found that histone crotonylation can inhibit the expression of pro-growth genes [43] and sex chromosome-linked genes [44]. Recently, although there are more and more articles reporting the crotonylation of non-histone proteins, the scope of these studies is not very broad, and there is no report in cardiovascular field. However, the crotonylation of lysine in nonhistone proteins in VSMCs is not reported so far.

To identify whether lysine crotonylation occurs in VSMCs and to explore its possible function in cellular bioprocesses, a comprehensive crotonyl-omics was realized by combing high-resolution LC-MS/MS with highly sensitive immune-affinity purification. A total of 2138 lysine crotonylation sites distributed in 534 proteins were identified, representing the most abundant lysine acylation proteome reported in the nonhistone proteins of VSMCs. These crotonylated proteins were involved in multiple biological processes, including glucose metabolism, amino acids biosynthesis, protein folding, and RNA degradation according to $\mathrm{GO}$ and KEGG pathway analysis. The enrichment analysis also coordinated with the PPI network analysis which showed widespread interaction between crotonylated proteins and their function clustering, such as ribosome, spliceosome.

Lysine acetylation is abundant in many glucose-metabolic pathways, such as carbon metabolism, glycolysis/gluconeogenesis, the tricarboxylic acid (TCA) cycle, pentose phosphate pathway, and conservative in eukaryotes, also in prokaryotes [14,15,45-47]. In our paper, we found numerous enzymes that involved in the glucose-metabolic pathways could be modified on lysine by crotonyl. In PDGF-BB stimulated VSMCs, the enzymes that undergo crotonylation were mainly those involved in glycolysis. There were 50\% (8/16) enzymes in the glycolysis/gluconeogenesis that occur crotonylation at a great many sites, with an average of 10 . For example, Pyruvate kinase (PKM), one of the key glycolysis 
metabolism enzymes, was crotonylated at 16 different sites. The key lysine residue of PKM, K305 which was acetylated and could result in decreased PKM activity [48], also showed up-regulated crotonylation modification. This indicated that crotonylation possibly modulates the activity of PKM in cooperation and coordination with acetylation. Moreover, $30 \%(3 / 10)$ of the enzymes involved in pyruvate metabolism were modified by crotonylation and these enzymes have not been reported on acetylation. These results suggested that lysine crotonylation of lysine might play an important role in the regulation glycolysis/gluconeogenesis metabolism.

VSMCs show significant phenotypic and functional changes after vascular injury. In presence of vascular injury, the contractile phenotype of VSMCs can convert to the proliferative phenotype, and contraction markers showed a decrease accompanied by this change. Other mediators of proinflammatory which could induce the proliferation and chemotaxis would gradually increase. Activated VSMCs could proliferate and migrate significantly thereby accelerating post-injury repair of blood vessels. In the present study, lysine crotonylation of 5 VSMC contractile markers extensively occurred at multiple sites. For example, caldesman 1 was crotonylated at 16 sites and myosin 9 at 15 sites. Moreover, transgelin was also crotonylated at 3 lysine sites. These results suggest that lysine crotonylation are likely involved in regulating VSMC contraction.

Proteins are biological macromolecules that perform a wide range of functions in living organisms. Amino acids are the basic units of protein. Their synthesis and degradation were related to the lysine crotonylation showing in our research, such as arginine, proline, cysteine, and methionine. Protein synthesis was also affected by lysine crotonylation. In our study, we found 21 ribosome subunits that were crotonylated. The crotonylated ribosome proteins participate in the stabilization of the structure and the formation of peptides. Shashi Bhushan. et al demonstrated that nascent polypeptide chains are connected in a set of discrete tunnel components, including rRNA nucleotides U2585, A2062, A2058, A751, and the extensions of L4 and L17 (L22) [49]. And, the L4 globular domain promoted the folding of early rRNA, and the internal loop could aggregate and stabilize the domain of the $60 \mathrm{~S}$ subunit functional active site [50]. Also, S3 could bind to receptor for activated c-kinase (RACK1, Asc1 in yeast) through the C-terminal extension sequence to stabilize protein synthesis and cell growth [51]. HSP60 and HSP90 family are chaperones which give assistance to the amino acid folding progress. They were extensively crotonylated after VSMCs activating and this result indicated that lysine crotonylation might affect the folding process. In the present study, we found ubiquitin-40S ribosomal protein S27a (Rps27a) was modified through crotonylation. These results thus imply that crotonylation of lysine is involved in the regulation of protein synthesis, folding process, and ubiquitination degradation.

Together with crotonylome, nonhistone proteins ubiquitylnome of VSMCs was performed by LC-MS/MS accompanied by highly sensitive immune-affinity purification. The results showed a total of 1359 quantitative sites with 657 proteins. These ubiquitinated proteins were mainly involved in cell morphogenesis, cytoskeleton and cellular component organization, proteasome, ribosome, and regulation of actin cytoskeleton based on the analysis of GO and KEGG pathway. 
In the past research, there are articles summarizing the crosstalk of multiple post-translational modifications in Candida albicans [52], developing rice seeds [53], yeast sporulation and mouse spermatogenesis [54]. In VSMC, little is known about the crosstalk of multi-post-translational modifications. In our work, we found some proteins and function overlap in crotonylation and ubiqutination. The results of the crosstalk analysis showed that 199 sites of 177 proteins could modified by crotonyl and ubiquitin simultaneously. The obviously changeable GO and KEGG pathway included cytoskeleton, cytoskeleton protein binding, actin binding and regulation of actin cytoskeleton. These cellular component and function analysis indicated that crotonylation and ubiquitination were involved in cytoskeleton remodeling and modulated the phenotypic transformation. In the PPI network analysis with function clustering, we found MYH9 (myosin heavy chain 9), containing upregulated 18 crotonylated lysine sites and downregulated 3 ubiquitinated sites, was the point of intersection of tight junction, VSMCs contraction and regulation of actin cytoskeleton. MYH9 is a widespread cytoplasmic myosin and involved in translocation of the skeleton proteins [55]. Moreover, some studies showed that MYH9 was upregulated in gastric cancer which could promote the transcription of $\beta$-catenin [56] and upregulated in esophageal squamous cell carcinoma (ESCC) facilitating cell metastasis [57]. Also, MYH9 could bind with and degrade GSK3 $\beta$ through ubiquitin, therefore down regulated $\beta$-catenin to induce the epithelialmesenchymal transition in hepatocellular carcinoma [58]. However, the function of MYH9 in phenotypic remodeling remains unknown which provides a possible direction for subsequent research.

In our previous study, the activity of G6PD could be affected by SM22a ubiquitination [13], which indicated the possible relation of PTM and phenotypic remodeling. Combining with the enrichment analysis of GO, KEGG pathway and PPI network, we found that enzymes of the glucose metabolism pathways including glycolysis/gluconeogenesis, pentose phosphate pathway, pyruvate metabolism and TCA cycle, were modified extensively by crotonyl and ubiquitin. The modification of the enzymes might modulate the pathways directly or indirectly. Among these pathways, the enzymes of glycolysis were the mostly both crotonylated and ubiquitinated. Meanwhile in other pathways, crotonylation is more widely distributed, suggesting its significance on the modulation of glycose metabolism in VSMCs.

\section{Conclusion}

In summary, our study gave an overview of the profiling analysis of global nonhistone proteins lysine crotonylome and ubiquitylome in VSMCs for the first time. The results showed more prevalence of crotonylation than ubiquitination and their crosstalk analysis focusing on GO, KEGG and PPI suggested that the biological significance of them were associated with glycolysis, VSMCs contraction or cellular skeleton modulation. The crosstalk of crotonylation and ubiquitination in glycolysis is possibly a novel mechanism underlying the VSMC phenotypic remodeling. Related crotonylated and ubiquitinated proteins will be the emphasis for further functional studies.

\section{Methods}

Experimental Procedures 


\section{Preparation of VSMC extraction for LC-MS}

VSMCs with or without PDGF-BB stimulated were sonicated three times on ice using a high intensity ultrasonic processor (Scientz) in lysis buffer (8 M urea, $3 \mu \mathrm{M}$ TSA, 50 mM NAM and 1\% Protease Inhibitor Cocktail). The remaining debris was removed by centrifugation at $12,000 \mathrm{~g}$ at $4{ }^{\circ} \mathrm{C}$ for $10 \mathrm{~min}$. Finally, the supernatant was collected and the protein concentration was determined with BCA kit according to the manufacturer's instructions.

\section{Trypsin Digestion}

For digestion, the protein solution was reduced with $5 \mathrm{mM}$ dithiothreitol for $30 \mathrm{~min}$ at $56^{\circ} \mathrm{C}$ and alkylated with $11 \mathrm{mM}$ iodoacetamide for $15 \mathrm{~min}$ at room temperature in darkness. The protein sample was then diluted by adding $100 \mathrm{mM} \mathrm{NH}_{4} \mathrm{HCO}_{3}$ to urea concentration less than $2 \mathrm{M}$. Finally, trypsin was added at 1:50 trypsin-to-protein mass ratio for the first digestion overnight and 1:100 trypsin-to-protein mass ratio for a second $4 \mathrm{~h}$-digestion.

\section{HPLC Fractionation}

The tryptic peptides were fractionated into fractions by high $\mathrm{pH}$ reverse-phase HPLC using Thermo Betasil C18 column ( $5 \mu \mathrm{m}$ particles, $10 \mathrm{~mm}$ ID, $250 \mathrm{~mm}$ length). Briefly, peptides were first separated with a gradient of $8 \%$ to $32 \%$ acetonitrile ( $\mathrm{pH} \mathrm{9.0)} \mathrm{over} 60$ min into 60 fractions. Then, the peptides were combined into 10 fractions and dried by vacuum centrifuging.

\section{Affinity Enrichment of crotonylated peptides}

To enrich crotonylated peptides, tryptic peptides dissolved in NETN buffer (100 mM NaCl, $1 \mathrm{mM}$ EDTA, 50 $\mathrm{mM}$ Tris- $\mathrm{HCl}, 0.5 \% \mathrm{NP}-40, \mathrm{pH} 8.0$ ) were incubated with pre-washed antibody beads (PTM Bio) at $4^{\circ} \mathrm{C}$ overnight with gentle shaking. Then the beads were washed four times with NETN buffer and twice with $\mathrm{H}_{2} \mathrm{O}$. The bound peptides were eluted from the beads with $0.1 \%$ trifluoroacetic acid. Finally, the eluted fractions were combined and vacuum-dried. For LC-MS/MS analysis, the resulting peptides were desalted with C18 ZipTips (Millipore) according to the manufacturer's instructions.

\section{LC-MS/MS Analysis}

The tryptic peptides were dissolved in $0.1 \%$ formic acid (solvent $A$ ), directly loaded onto a home-made reversed-phase analytical column (15-cm length, $75 \mu \mathrm{m}$ i.d.). The gradient was comprised of an increase from $6 \%$ to $23 \%$ solvent B ( $0.1 \%$ formic acid in $98 \%$ acetonitrile) over $26 \mathrm{~min}, 23 \%$ to $35 \%$ in $8 \mathrm{~min}$ and climbing to $80 \%$ in 3 min then holding at $80 \%$ for the last $3 \mathrm{~min}$, all at a constant flow rate of $400 \mathrm{~nL} / \mathrm{min}$ on an EASY-nLC 1000 UPLC system. The peptides were subjected to NSI source followed by tandem mass spectrometry (MS/MS) in Q ExactiveTM Plus (Thermo) coupled online to the UPLC. The electrospray voltage applied was $2.0 \mathrm{kV}$. The $\mathrm{m} / \mathrm{z}$ scan range was 350 to 1800 for full scan, and intact peptides were detected in the Orbitrap at a resolution of 70,000. Peptides were then selected for MS/MS using NCE setting as 28 , and the fragments were detected in the Orbitrap at a resolution of 17,500 . A 
data-dependent procedure that alternated between one MS scan followed by $20 \mathrm{MS} / \mathrm{MS}$ scans with 15.0 s dynamic exclusion. Automatic gain control (AGC) was set at $5 E 4$.

\section{Database Search}

The resulting MS/MS data were processed using Maxquant search engine (v.1.5.2.8). Tandem mass spectra were searched against UniProt database concatenated with reverse decoy database. Trypsin/P was specified as cleavage enzyme, allowing up to 4 missing cleavages. The mass tolerance for precursor ions was set as 20 ppm in First search and 5 ppm in Main search, and the mass tolerance for fragment ions was set as 0.02 Da. Carbamidomethyl on Cys was specified as fixed modification and contractile and oxidation on Met were specified as variable modifications. Label-free quantification method was LFQ, FDR was adjusted to $<1 \%$ and the minimum score for modified peptides was set $>40$.

\section{Cell culture and treatment}

VSMC was isolated from the thoracic aorta of 80-100 g male Sprague-Dawley rats as previously described. VSMCs were grown in low glucose Dulbecco's-modified Eagle's medium (DMEM) (Invitrogen, US) with $10 \%$ fetal bovine serum (FBS), $100 \mathrm{U} / \mathrm{ml}$ pencillin, and $100 \mathrm{ug} / \mathrm{ml}$ streptomycin. The VSMCs were maintained at $37^{\circ} \mathrm{C}$ in a humidified atmosphere containing $5 \% \mathrm{CO}_{2}$, and only passages 3 to 5 cells at $70-80 \%$ confluence were used in the experiments, except if stated otherwise. HEK293 cells were cultured in high glucose DMEM containing 10\% FBS. This study was performed via a protocol approved by the Institutional Animal Care and Use Committee of Hebei Medical University, in accordance with the Guide for the Care and Use of Laboratory Animals, and the Hebei Medical University Clinical Research Ethics Committee.

\section{Plasmids and antibodies}

The cDNA of PKM and LDHA were amplified by PCR and subcloned into pcDNA-HA vector (biyuntian). The following antibodies were used in the expriments: Pan-Kcr (PTM Biol 501), FLAG and HA.

\section{Co-immunoprecipitation}

Cells were lysed in NP40 buffer (50 mM Tris-HCl, pH 7.4, 150 mM NaCl, 1\% NP-40, 1 mM EDTA, 10 mM sodium butyrate) containing protease inhibitors for 30 min on ice. Then, lysates were incubated with relevant antibody overnight at $4{ }^{\circ} \mathrm{C}$. And $50 \mu$ protein $A / G$ agarose beads were added and incubated for 2 h at $4{ }^{\circ} \mathrm{C}$. After washed with NP40 buffer for three times, the immunoprecipitated complexes were subjected to SDS-PAGE and immunoblotted with the indicated antibodies.

\section{Immunofluorescence}

Cells were cultivated on coverslips prior to experiment. Cells were pre-washing with PBS for twice and fixed with $4 \%$ paraformaldehyde for $15 \mathrm{~min}$ at room temperature. Then the cells were permeabilized with 0.1\% NP-40 for 15 min. Permeabilized cells were blocked with 5\% BSA in PBS for $1 \mathrm{~h}$. After washed with 
PBS three times, cells were incubated overnigt at $4{ }^{\circ} \mathrm{C}$ with pan-Kcr antibody. After washed with PBS for three times, cells were incubated with FITC-goat anti-rabbit IgG for $1 \mathrm{~h}$ at $4{ }^{\circ} \mathrm{C}$. Finally, cells were stained with DAPI and mounted to glass slides. Microscopic imaged were captured by confocal laser-scanning microscope (Leica).

\section{Declarations}

\section{Ethics approval and consent to participate}

Not applicable.

\section{Consent for publication}

All authors agree to publish this paper.

\section{Availability of data and materials}

All data generated or analyzed during this study are included in this published article and its Additional files. The datasets on original profiling of VSMCs are not publicly available due to potential conflicts of interests, but are available from the corresponding author on reasonable request.

\section{Competing interests}

The authors have no competing interests to disclose.

\section{Funding}

This study was supported by grants from the National Natural Science Foundation of China (81670394), the Hebei Natural Science Foundation (C2019206022) and the Hebei Scientific and Technological Research Projects (ZD2020302).

\section{Authors' contributions}

Cao contributed to organize and analyze the data, make the figures, and write the most of manuscript. Chen wrote the part of ubiquitination. Yue, Jiang, Ma contributed to organize the data and figures. Dong contributed to analyze and interpret the data, to write and revise the manuscript, and obtain the funding.

\section{Acknowledgements}

The authors thank Jingjie PTM Biolab Co. Ltd. (Hangzhou, China) for the performance of LC-MS/MS.

\section{References}

1. Andersson C, Vasan RS. Epidemiology of cardiovascular disease in young individuals. Nat Rev Cardiol. 2018;15(4):230-40. 
2. Petsophonsakul P, Furmanik M, Forsythe R, Dweck M, Schurink GW, Natour E, et al. Role of Vascular Smooth Muscle Cell Phenotypic Switching and Calcification in Aortic Aneurysm Formation. Arterioscler Thromb Vasc Biol. 2019;39(7):1351-68.

3. Gomez D, Owens GK. Smooth muscle cell phenotypic switching in atherosclerosis. Cardiovasc Res. 2012;95(2):156-64.

4. Owens GK, Kumar MS, Wamhoff BR. Molecular regulation of vascular smooth muscle cell differentiation in development and disease. Physiol Rev. 2004;84(3):767-801.

5. Kim DN, Schmee J, Baker JE, Lunden GM, Sheehan CE, Lee CS, et al. Dietary fish oil reduces microthrombi over atherosclerotic lesions in hyperlipidemic swine even in the absence of plasma cholesterol reduction. Exp Mol Pathol. 1993;59(2):122-35.

6. Cercek B, Sharifi B, Barath P, Bailey L, Forrester JS. Growth factors in pathogenesis of coronary arterial restenosis. Am J Cardiol. 1991;68(12):24C-33C.

7. Thyberg J, Ostman A, Backstrom G, Westermark B, Heldin CH. Localization of platelet-derived growth factor (PDGF) in CHO cells transfected with PDGF A- or B-chain CDNA: retention of PDGF-BB in the endoplasmic reticulum and Golgi complex. J Cell Sci. 1990;97 (Pt 2):219-29.

8. Heldin $\mathrm{CH}$, Westermark B. Signal transduction by the receptors for platelet-derived growth factor. J Cell Sci. 1990;96 (Pt 2):193-6.

9. Kaplan-Albuquerque N, Van Putten V, Weiser-Evans MC, Nemenoff RA. Depletion of serum response factor by RNA interference mimics the mitogenic effects of platelet derived growth factor-BB in vascular smooth muscle cells. Circ Res. 2005;97(5):427-33.

10. Cain JA, Solis N, Cordwell SJ. Beyond gene expression: the impact of protein post-translational modifications in bacteria. J Proteomics. 2014;97:265-86.

11. Olsen JV, Mann M. Status of large-scale analysis of post-translational modifications by mass spectrometry. Mol Cell Proteomics. 2013;12(12):3444-52.

12. Zhao Y, Jensen ON. Modification-specific proteomics: strategies for characterization of posttranslational modifications using enrichment techniques. Proteomics. 2009;9(20):4632-41.

13. Dong LH, Li L, Song Y, Duan ZL, Sun SG, Lin YL, et al. TRAF6-Mediated SM22alpha K21

Ubiquitination Promotes G6PD Activation and NADPH Production, Contributing to GSH Homeostasis and VSMC Survival In Vitro and In Vivo. Circ Res. 2015;117(8):684-94.

14. Zhao S, Xu W, Jiang W, Yu W, Lin Y, Zhang T, et al. Regulation of cellular metabolism by protein lysine acetylation. Science. 2010;327(5968):1000-4. 
15. Kim SC, Sprung R, Chen Y, Xu Y, Ball H, Pei J, et al. Substrate and functional diversity of lysine acetylation revealed by a proteomics survey. Mol Cell. 2006;23(4):607-18.

16. Xie Z, Dai J, Dai L, Tan M, Cheng Z, Wu Y, et al. Lysine succinylation and lysine malonylation in histones. Mol Cell Proteomics. 2012;11(5):100-7.

17. Nakamura N. Ubiquitin System. Int J Mol Sci. 2018;19(4).

18. Peng C, Lu Z, Xie Z, Cheng Z, Chen Y, Tan M, et al. The first identification of lysine malonylation substrates and its regulatory enzyme. Mol Cell Proteomics. 2011;10(12):M111-12658.

19. Tan M, Luo H, Lee S, Jin F, Yang JS, Montellier E, et al. Identification of 67 histone marks and histone lysine crotonylation as a new type of histone modification. Cell. 2011;146(6):1016-28.

20. Yang XJ, Seto E. Lysine acetylation: codified crosstalk with other posttranslational modifications. Mol Cell. 2008;31(4):449-61.

21. Sabari BR, Tang Z, Huang H, Yong-Gonzalez V, Molina H, Kong HE, et al. Intracellular crotonyl-CoA stimulates transcription through p300-catalyzed histone crotonylation. Mol Cell. 2015;58(2):203-15.

22. Madsen AS, Olsen CA. Profiling of substrates for zinc-dependent lysine deacylase enzymes: HDAC3 exhibits decrotonylase activity in vitro. Angew Chem Int Ed Engl. 2012;51(36):9083-7.

23. Zhao $D$, Guan $H$, Zhao S, Mi W, Wen H, Li Y, et al. YEATS2 is a selective histone crotonylation reader. Cell Res. 2016;26(5):629-32.

24. Bao X, Wang Y, Li X, Li XM, Liu Z, Yang T, et al. Identification of 'erasers' for lysine crotonylated histone marks using a chemical proteomics approach. Elife. 2014;3.

25. Feldman JL, Baeza J, Denu JM. Activation of the protein deacetylase SIRT6 by long-chain fatty acids and widespread deacylation by mammalian sirtuins. J Biol Chem. 2013;288(43):31350-6.

26. Palacios OM, Carmona JJ, Michan S, Chen KY, Manabe Y, Ward JR, et al. Diet and exercise signals regulate SIRT3 and activate AMPK and PGC-1alpha in skeletal muscle. Aging (Albany NY). 2009;1(9):77183.

27. Zhu J, Dong Q, Dong C, Zhang X, Zhang H, Chen Z. Global Lysine Crotonylation Alterations of Host Cell Proteins Caused by Brucella Effector BspF. Front Cell Infect Microbiol. 2020;10:603457.

28. Liu JF, Wu SF, Liu S, Sun X, Wang XM, Xu P, et al. Global Lysine Crotonylation Profiling of Mouse Liver. Proteomics. 2020;20(19-20):e2000049.

29. Chen W, Tang D, Xu Y, Zou Y, Sui W, Dai Y, et al. Comprehensive analysis of lysine crotonylation in proteome of maintenance hemodialysis patients. Medicine (Baltimore). 2018;97(37):e12035. 
30. Xu W, Wan J, Zhan J, Li X, He H, Shi Z, et al. Global profiling of crotonylation on non-histone proteins. Cell Res. 2017;27(7):946-9.

31. Liu M, Yan M, Lv H, Wang B, Lv X, Zhang H, et al. Macrophage K63-Linked Ubiquitination of YAP Promotes Its Nuclear Localization and Exacerbates Atherosclerosis. Cell Rep. 2020;32(5):107990.

32. Dang F, Nie L, Wei W. Ubiquitin signaling in cell cycle control and tumorigenesis. Cell Death \& Differentiation. 2021;28(2):427-38.

33. Chu Y, Kang Y, Yan C, Yang C, Zhang T, Huo H, et al. LUBAC and OTULIN regulate autophagy initiation and maturation by mediating the linear ubiquitination and the stabilization of ATG13. Autophagy. 2021;17(7):1684-99.

34. Dong LH, Wen JK, Miao SB, Jia Z, Hu HJ, Sun RH, et al. Baicalin inhibits PDGF-BB-stimulated vascular smooth muscle cell proliferation through suppressing PDGFRbeta-ERK signaling and increase in p27 accumulation and prevents injury-induced neointimal hyperplasia. Cell Res. 2010;20(11):1252-62.

35. Yin LM, Schnoor M, Jun CD. Structural Characteristics, Binding Partners and Related Diseases of the Calponin Homology (CH) Domain. Front Cell Dev Biol. 2020;8:342.

36. Xie X, Kang H, Liu W, Wang GL. Comprehensive profiling of the rice ubiquitome reveals the significance of lysine ubiquitination in young leaves. J Proteome Res. 2015;14(5):2017-25.

37. Guo J, Liu J, Wei Q, Wang R, Yang W, Ma Y, et al. Proteomes and Ubiquitylomes Analysis Reveals the Involvement of Ubiquitination in Protein Degradation in Petunias. Plant Physiol. 2017;173(1):668-87.

38. Danielsen JM, Sylvestersen KB, Bekker-Jensen S, Szklarczyk D, Poulsen JW, Horn H, et al. Mass spectrometric analysis of lysine ubiquitylation reveals promiscuity at site level. Mol Cell Proteomics. 2011;10(3):M110-3590.

39. Dong LH, Li L, Song Y, Duan ZL, Sun SG, Lin YL, et al. TRAF6-Mediated SM22alpha K21 Ubiquitination Promotes G6PD Activation and NADPH Production, Contributing to GSH Homeostasis and VSMC Survival In Vitro and In Vivo. Circ Res. 2015;117(8):684-94.

40. Harris AR, Belardi B, Jreij P, Wei K, Shams H, Bausch A, et al. Steric regulation of tandem calponin homology domain actin-binding affinity. Mol Biol Cell. 2019;30(26):3112-22.

41. Xiong W, Tang T, Littleton E, Karcini A, Lazar IM, Capelluto DGS. Preferential phosphatidylinositol 5phosphate binding contributes to a destabilization of the VHS domain structure of Tom1. Sci Rep-Uk. 2019;9(1).

42. Lohi O, Poussu A, Mao Y, Quiocho F, Lehto V. VHS domain - a longshoreman of vesicle lines. Febs Lett. 2002;513(1):19-23. 
43. Gowans GJ, Bridgers JB, Zhang J, Dronamraju R, Burnetti A, King DA, et al. Recognition of Histone Crotonylation by Taf14 Links Metabolic State to Gene Expression. Mol Cell. 2019;76(6):909-21.

44. Liu S, Yu H, Liu Y, Liu X, Zhang Y, Bu C, et al. Chromodomain Protein CDYL Acts as a Crotonyl-CoA Hydratase to Regulate Histone Crotonylation and Spermatogenesis. Mol Cell. 2017;67(5):853-66.

45. Choudhary C, Kumar C, Gnad F, Nielsen ML, Rehman M, Walther TC, et al. Lysine acetylation targets protein complexes and co-regulates major cellular functions. Science. 2009;325(5942):834-40.

46. Finkemeier I, Laxa M, Miguet L, Howden AJ, Sweetlove LJ. Proteins of diverse function and subcellular location are lysine acetylated in Arabidopsis. Plant Physiol. 2011;155(4):1779-90.

47. He D, Wang Q, Li M, Damaris RN, Yi X, Cheng Z, et al. Global Proteome Analyses of Lysine Acetylation and Succinylation Reveal the Widespread Involvement of both Modification in Metabolism in the Embryo of Germinating Rice Seed. J Proteome Res. 2016;15(3):879-90.

48. Lv L, Li D, Zhao D, Lin R, Chu Y, Zhang H, et al. Acetylation targets the M2 isoform of pyruvate kinase for degradation through chaperone-mediated autophagy and promotes tumor growth. Mol Cell. 2011;42(6):719-30.

49. Bhushan S, Meyer H, Starosta AL, Becker T, Mielke T, Berninghausen O, et al. Structural basis for translational stalling by human cytomegalovirus and fungal arginine attenuator peptide. Mol Cell. 2010;40(1):138-46.

50. Gamalinda M, Woolford JJ. Deletion of L4 domains reveals insights into the importance of ribosomal protein extensions in eukaryotic ribosome assembly. Rna. 2014;20(11):1725-31.

51. Singh N, Jindal S, Ghosh A, Komar AA. Communication between RACK1/Asc1 and uS3 (Rps3) is essential for RACK1/Asc1 function in yeast Saccharomyces cerevisiae. Gene. 2019;706:69-76.

52. Zhou X, Song N, Li D, Li X, Liu W. Systematic Analysis of the Lysine Crotonylome and Multiple Posttranslational Modification Analysis (Acetylation, Succinylation, and Crotonylation) in Candida albicans. Msystems. 2021;6(1).

53. Meng X, Mujahid H, Zhang Y, Peng X, Redoña ED, Wang C, et al. Comprehensive Analysis of the Lysine Succinylome and Protein Co-modifications in Developing Rice Seeds. Mol Cell Proteomics. 2019;18(12):2359-72.

54. Crespo M, Damont A, Blanco M, Lastrucci E, Kennani SE, laly-Radio C, et al. Multi-omic analysis of gametogenesis reveals a novel signature at the promoters and distal enhancers of active genes. Nucleic Acids Res. 2020;48(8):4115-38.

55. Pecci A, Ma X, Savoia A, Adelstein RS. MYH9: Structure, functions and role of non-muscle myosin IIA in human disease. Gene. 2018;664:152-67. 
56. Ye G, Yang Q, Lei X, Zhu X, Li F, He J, et al. Nuclear MYH9-induced CTNNB1 transcription, targeted by staurosporin, promotes gastric cancer cell anoikis resistance and metastasis. Theranostics. 2020;10(17):7545-60.

57. Yang B, Liu H, Bi Y, Cheng C, Li G, Kong P, et al. MYH9 promotes cell metastasis via inducing Angiogenesis and Epithelial Mesenchymal Transition in Esophageal Squamous Cell Carcinoma. Int $\mathrm{J}$ Med Sci. 2020;17(13):2013-23.

58. Lin X, Li AM, Li YH, Luo RC, Zou YJ, Liu YY, et al. Silencing MYH9 blocks HBx-induced GSK3beta ubiquitination and degradation to inhibit tumor stemness in hepatocellular carcinoma. Signal Transduct Target Ther. 2020;5(1):13.

\section{Figures}


a
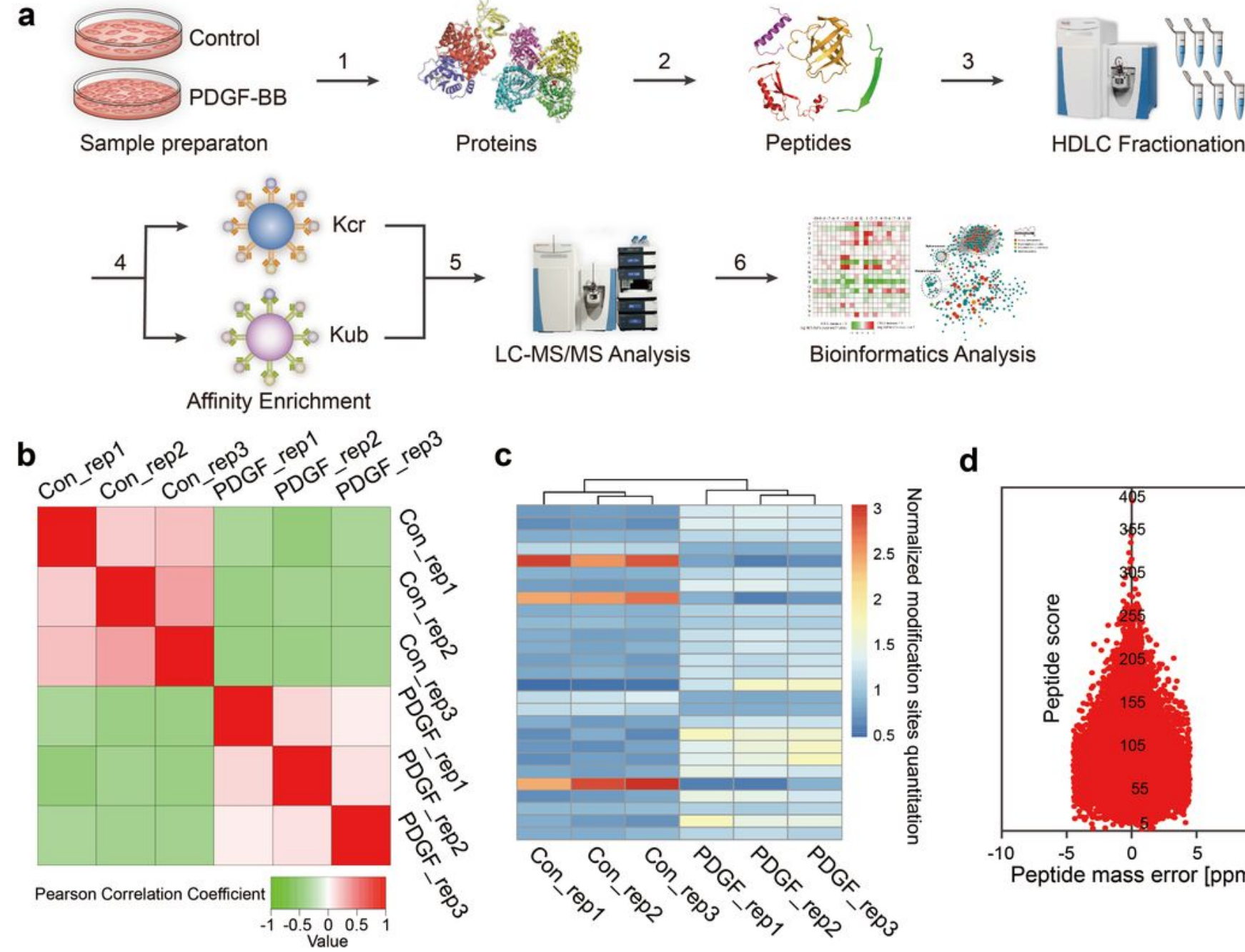

C

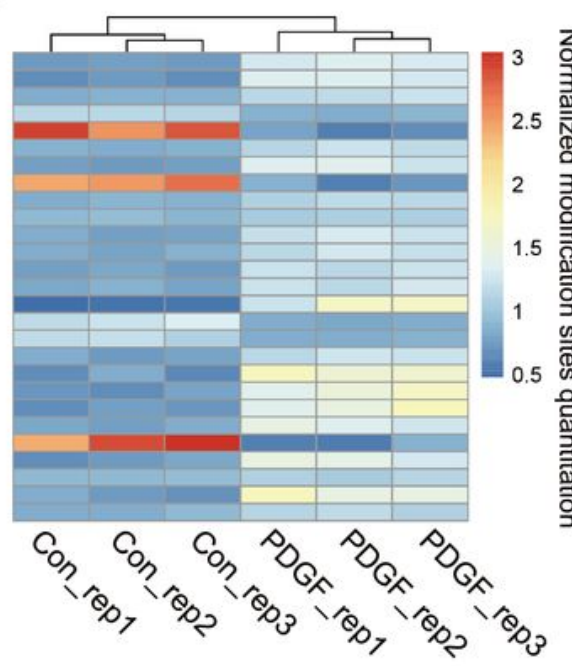

d

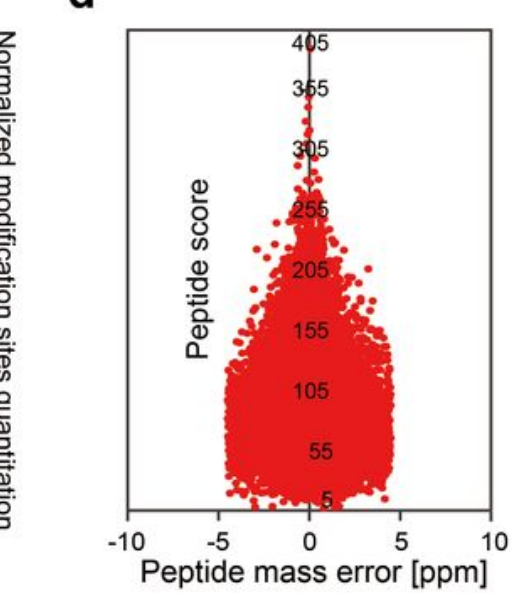

g

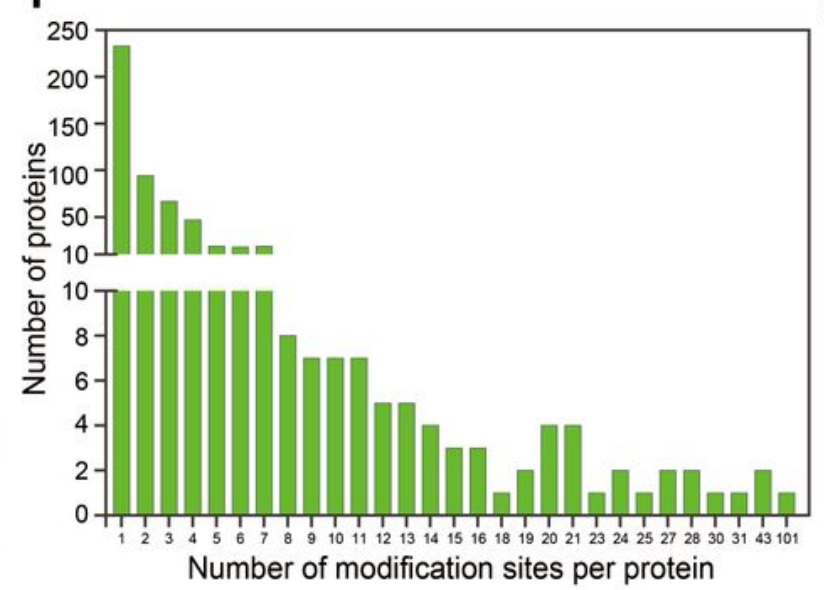

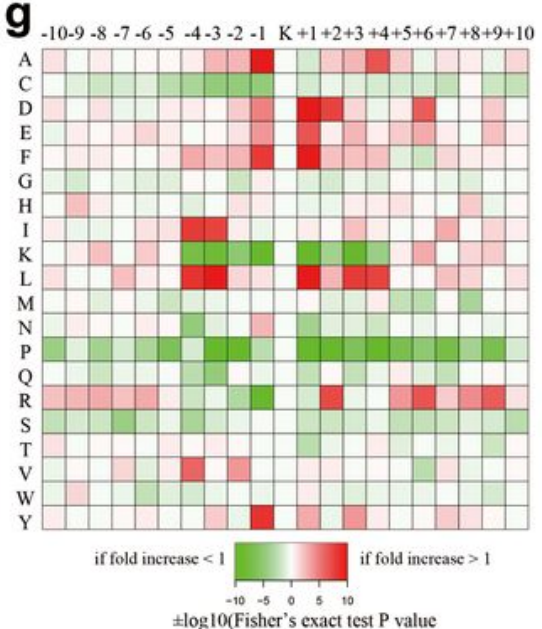

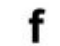

e

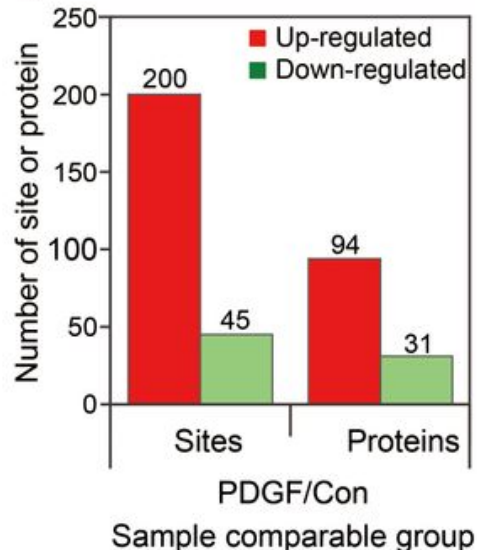

HDLC Fractionation

\section{Figure 1}

Proteomic and crotonylomic profiling showed significant differences in VSMCs upon PDGF-BB treatment and control group. a The systematic workflow for quantitative profiling of global crotonylome. VSMCs pre-and post-treatment with PDGF-BB were extracted (step 1) and digested with trypsin enzyme (step 2). High-performance liquid chromatography (HPLC) was used to separate the peptides (step 3), and we then analyzed the peptides with mass spectrometry (MS) (step 5) after affinity enrichment using the pan anti- 
crotonyl-lysine monoclonal antibody and the pan anti-ubiquitinate-lysine monoclonal antibody (step 4). Finally, we use the data from MS for bioinformatics analysis (step 6). b A heat map designed to calculate Pearson's Correlation Coefficient using pairings of all samples. This coefficient is a value to measure the degree of linear correlation between two sets of data: the closer Pearson coefficient is to -1 , the negative correlation is observed; the closer Pearson coefficient is to 1 , the positive correlation is observed; the closer Pearson coefficient is to 0 , the unrelated correlation is observed. In Figure $1 \mathrm{~b}$, red shows correlation coefficient of 1 , green shows correlation coefficient of -1 , and white shows a correlation coefficient of 0 . $c$ The heat map shows the quantitative differences in different modification sites between the two groups. $d$ The mass error of the whole identified peptides.e Differentially expressed proteins and modification sites in the PDGF group relative to the Con group. $f$ Statistical analysis of lysine crotonylation sites in all proteins. $g$ Motif enrichment heat map of all the upstream and downstream amino acids of the identified crotonylation modification sites. Con represents the group of control without PDGF-BB treatment, and PDGF represents the group with PDGF-BB treatment. Rep represents repeat sample. 


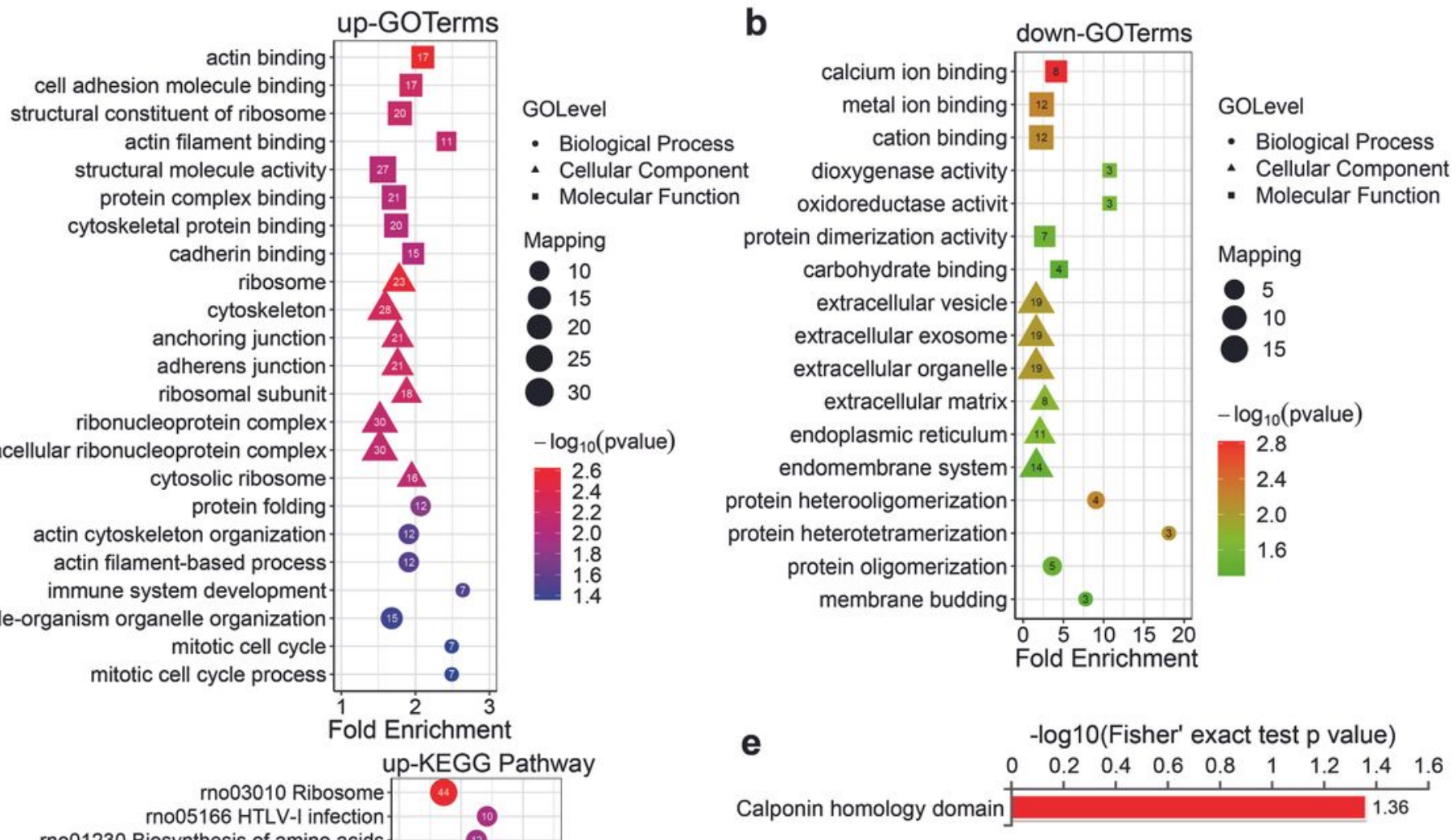

intracellular ribonucleoprotein complex
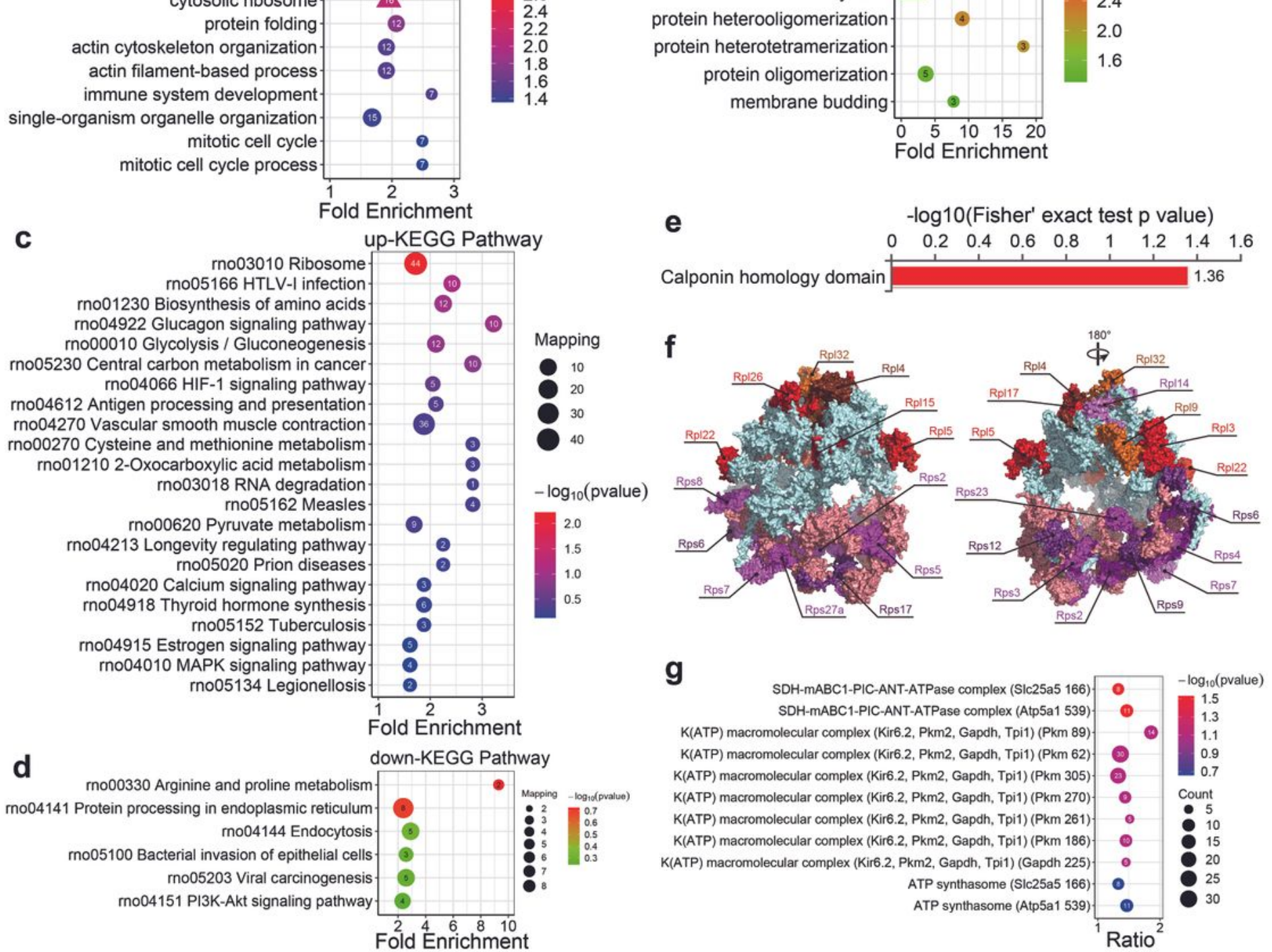

g

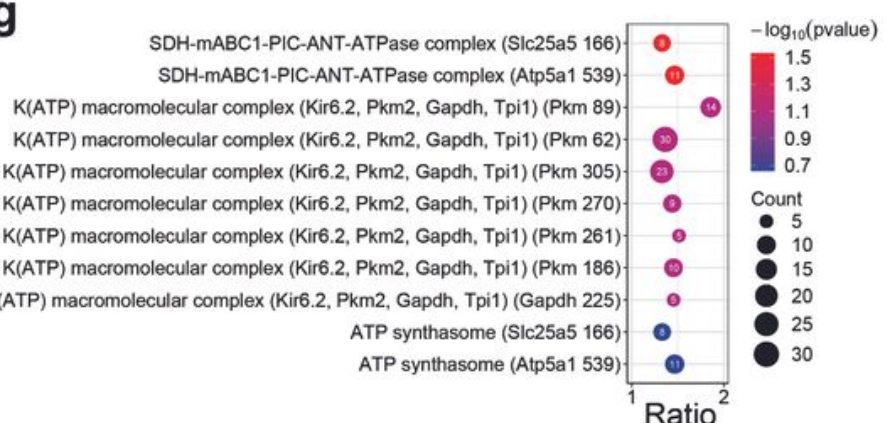

\section{Figure 2}

Enrichment analysis of the GO analysis, KEGG pathways and protein domains of the differentially modified sites. a The GO enrichment results of the protein corresponding to the up-regulated site and (b) the protein corresponding to the down-regulated site. c Enrichment analysis of KEGG pathway of upregulated crotonylated protein. $d$ Enrichment analysis of KEGG pathway of down-regulated crotonylated protein. e Enrichment analysis of protein domains of up-regulated crotonylated protein. $f$ The crystal 
structure of ribosome and the crotonylated ribosomal proteins. (PDB ID: 4V88) g Predicted functional enrichment of crotonylated protein complexes. The values on the horizontal axis are "Fold Enrichment" significant $p$-values $(p<0.05)$, which are converted to negative logarithms and then plotted as bubble charts. "pvalue" represents "Fisher' exact p value". Rpl: Ribosomal protein large subunit; Rps: Ribosomal protein small subunit.

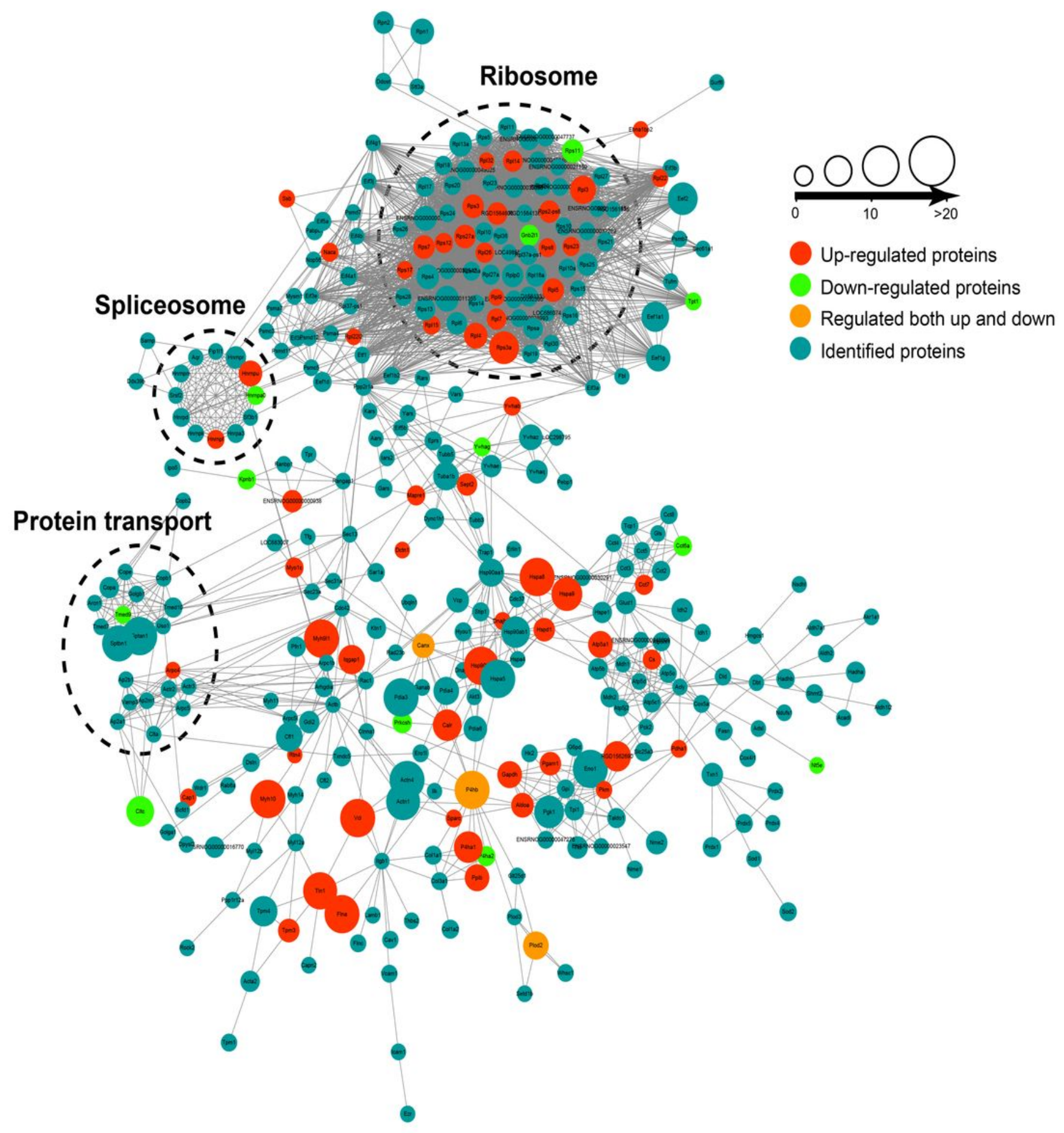

Figure 3 
Protein-protein interaction network was built according to the STRING database. Interactions belonging to the searched data set were selected.

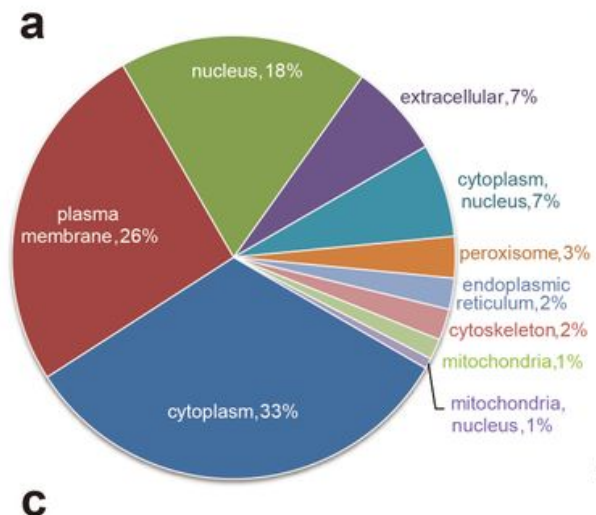

C

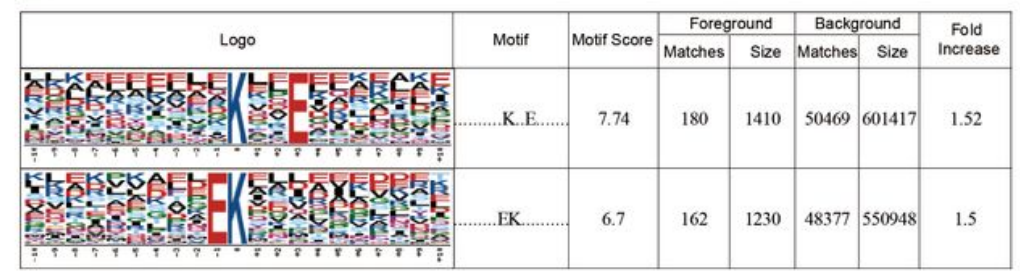

d

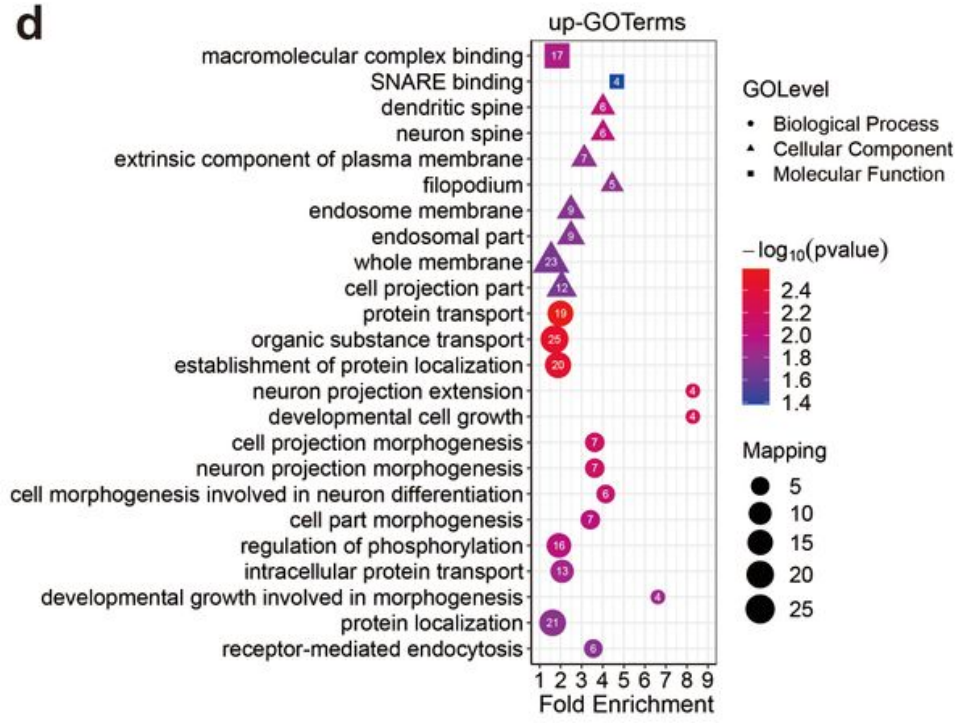

e

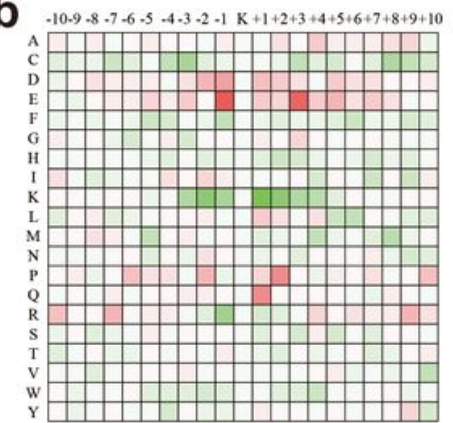
1

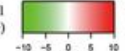

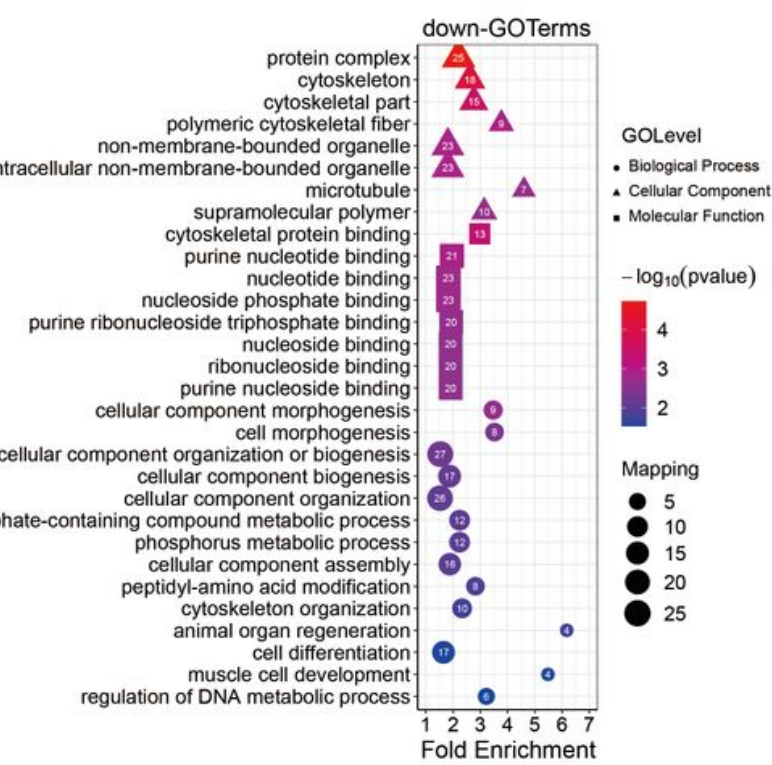

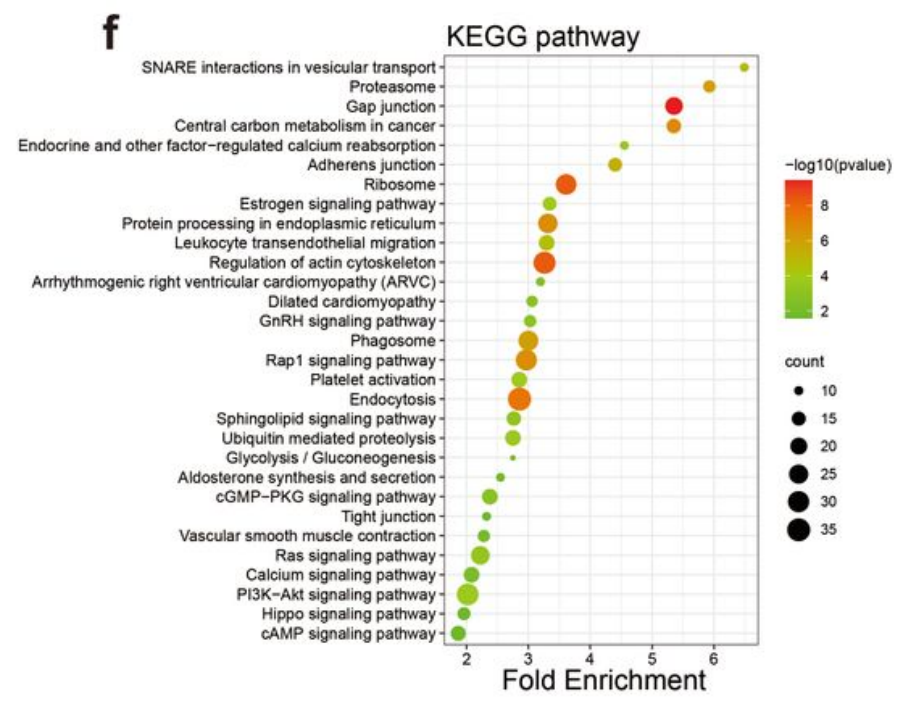

Figure 4

Properties of the quantified ubiquitylome. a The subcellular localization of the differentially ubiquitination modified proteins. $b$ Ubiquitination motifs and the conservation of the ubiquitinated sites. $c$ Heat map of the amino acid compositions of the lysine ubiquitination sites showing the frequency of different types of amino acids around the ubiquitinated lysine. d,e GO-based enrichment analysis of proteins with upregulated (d) and down-regulated (e) ubiquitination sites. $f$ KEGG pathway analysis. 
a

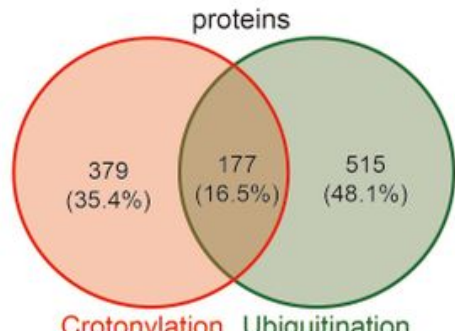

C Biological Process

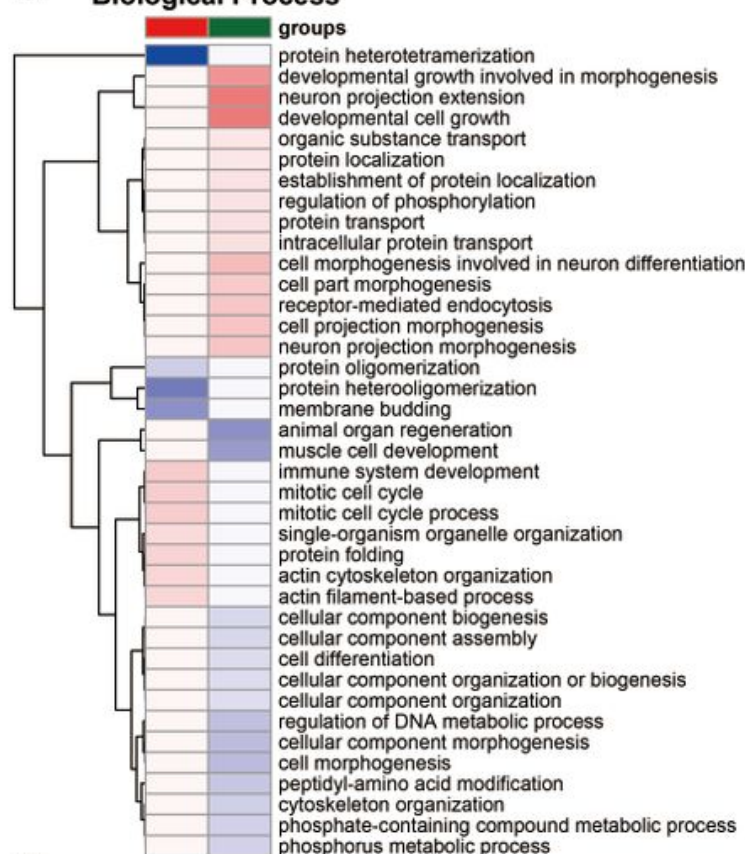

e

Molecular Function

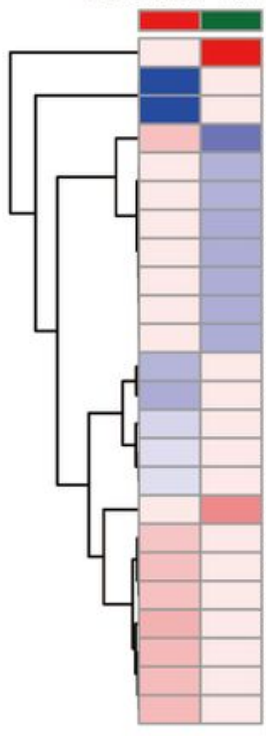

sites

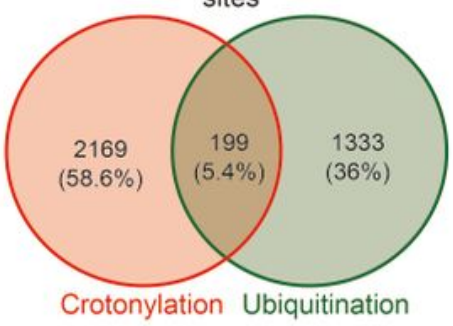

b Protein domain

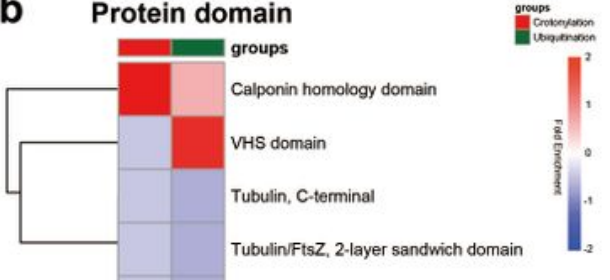

Tubulin/FtsZ, C-terminal

Tubulin/FtsZ, GTPase domain

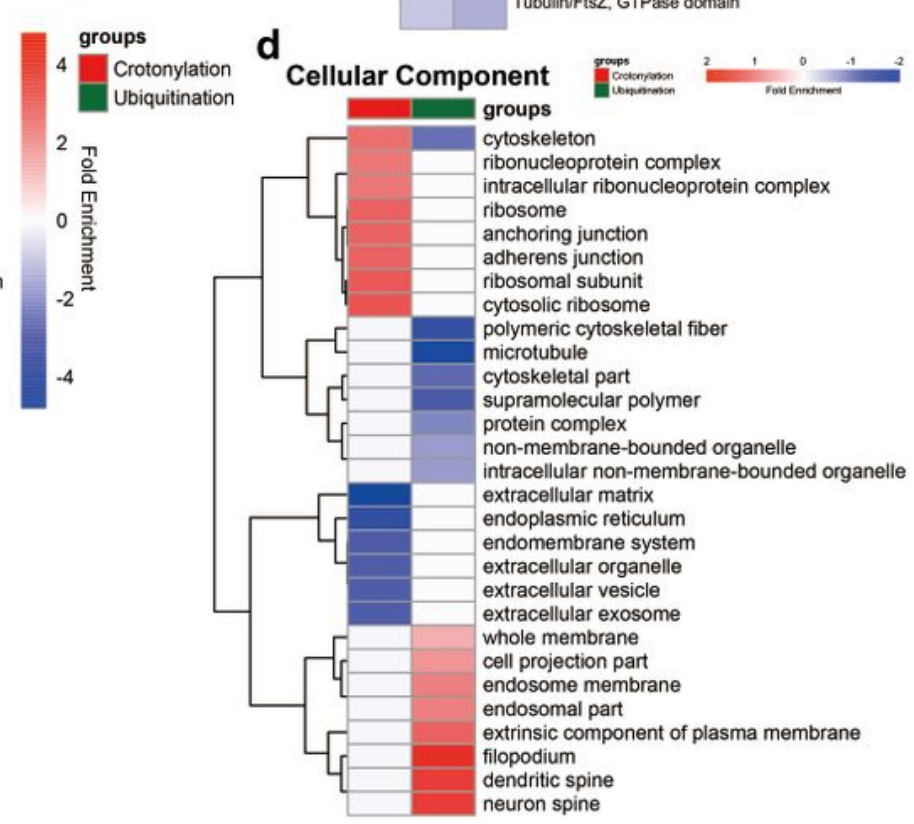

f

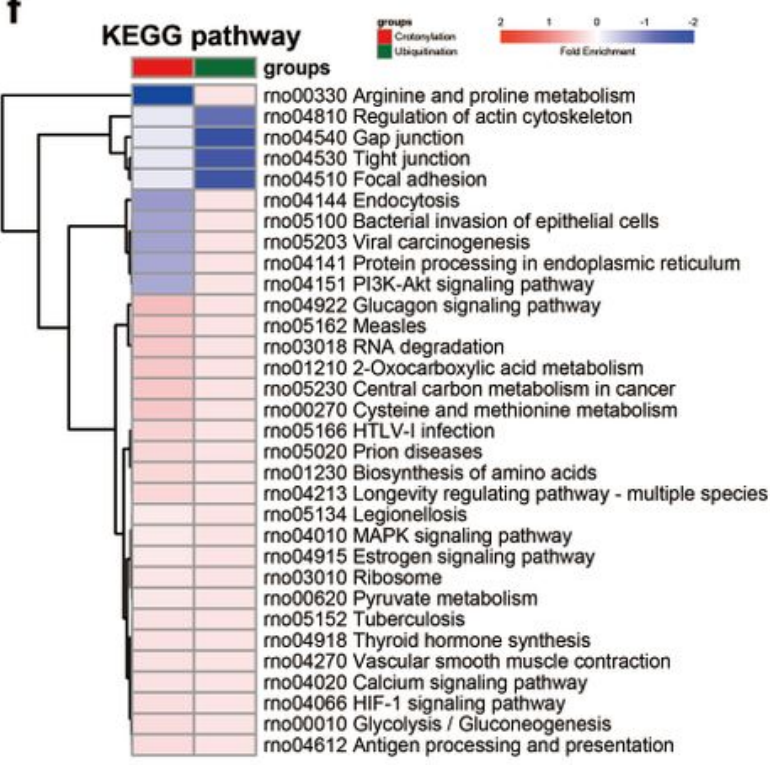

Figure 5

Crosstalk results of quantitative and enrichment analysis between crotonylation and ubiquitination. a The identified proteins and modification sites of lysine crotonylation and ubiqutination. b-f The heatmaps show the different and similar enrichment analysis of lysine crotonylation and ubiqutination, including protein domain (b), biological process (c), cellular component (d), molecular function (e) and KEGG pathway (f). 


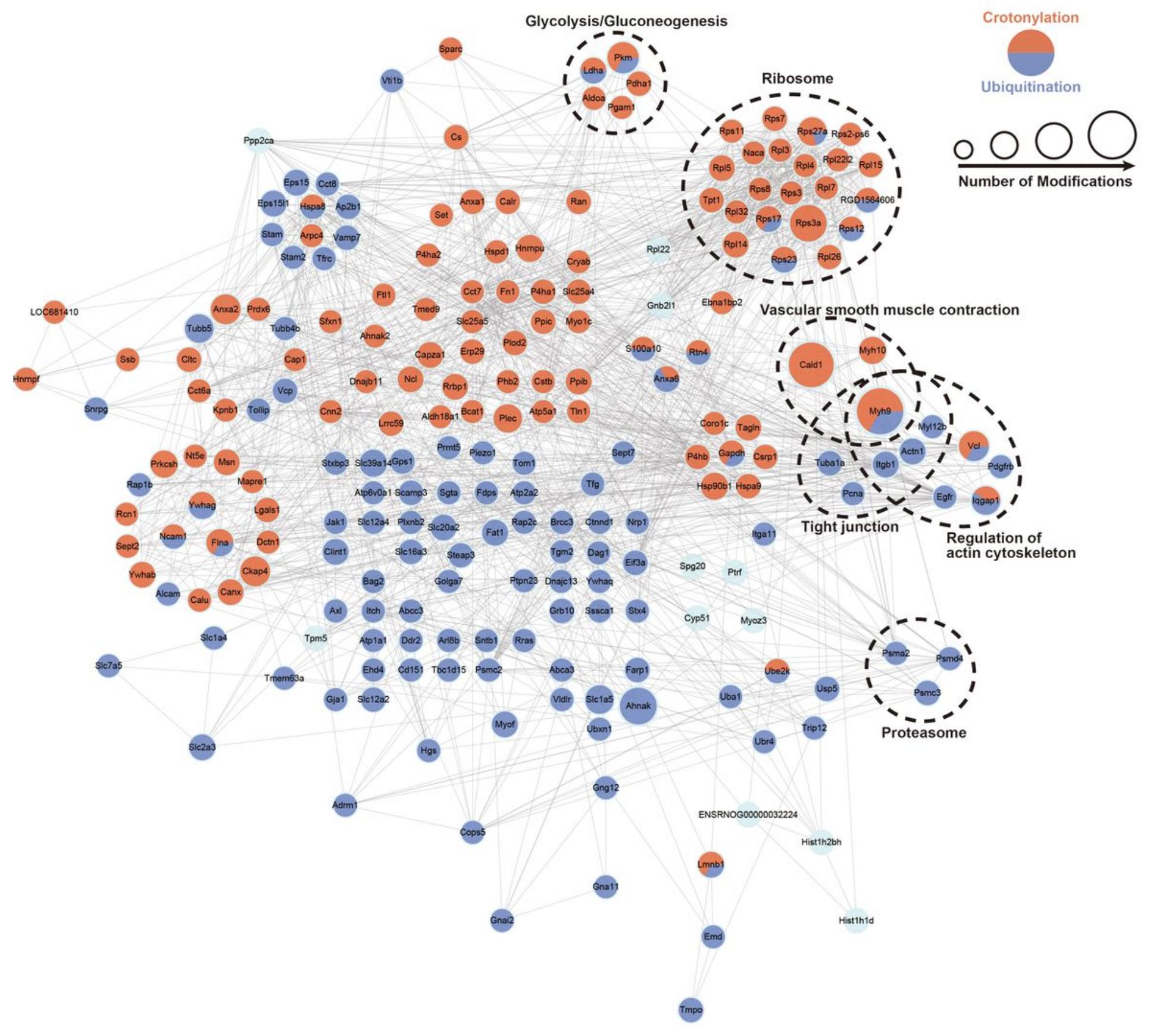

Figure 6

Protein-protein interaction network of crosstalk between crotonylation and ubiquitination was built according to the STRING database. The dotted circles show the function enrichment of the modified proteins. 


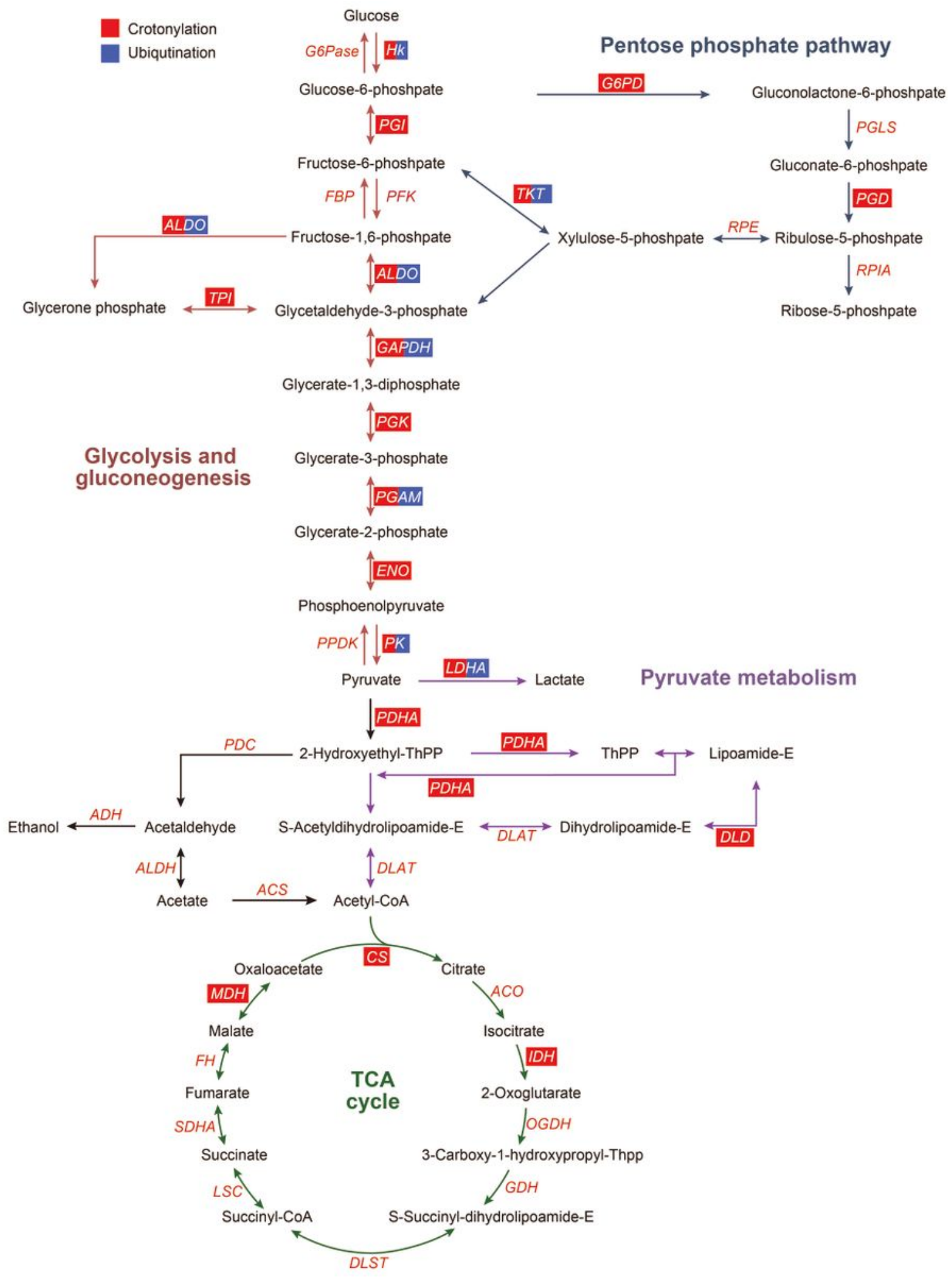

\section{Figure 7}

Crotonylated and ubiquitinated enzymes in glycolysis/gluconeogenesis, pentose phosphate pathway, pyruvate metabolism and TCA cycle. ACO: aconitate hydratase; ACS: acetyl coenzyme A synthetase; ADH: alcohol dehydrogena se; ALDH: aldehyde dehydrogenase; ALDO: aldolase; CS: citrate synthase; DLAT: dihydrolipoamide acetyltransferase (pyruvate dehydrogenase E2 component); DLD: dihydrolipoamide dehydrogenase; DLST: dihydrolipoamide succinyltransferase (2-oxoglutarate dehydrogenase E2 
component); ENO: enolase; FBP: fructose-biphosphatase; HK: hexokinase; IDH: isocitrate dehydrogenase; G6Pase: glucose-6-phosphatase; G6PD: glucose-6-phosphate dehydrogenase; GAPDH: glyceraldehyde phosphate dehydrogenase; LDHA: lactate dehydrogenase A; LSC: succinyl-CoA synthetase; MDH: malate dehydrogenase; OGDH: 2-oxoglutarate dehydrogenase $\mathrm{E} 1$ component; PDC: pyruvate dehydrogenase complex; PDH: pyruvate dehydrogenase; PFKA: phosphofructokinase; PK: pyruvate kinase; PGAM: phosphoglycerate mutase; PGD: 6-phosphogluconate dehydrogenase, decarboxylating 2; PGI: phosphohexose isomerase; PGK: phosphoglycerate kinase; PGLS: 6-phosphogluconolactonase; RPIA: ribose-5-phosphate isomerase; RPE: Ribulosephosphate 3-epimerase; TCA cycle: tricarboxylic acid cycle; SDHA: succinate dehydrogenase; TKT: transketolase; TPI: triose phosphate isomerase. The red box represents enzymes modified by crotonylation, and the blue box represent enzymes modified by ubiquitination.

\section{Supplementary Files}

This is a list of supplementary files associated with this preprint. Click to download.

- FigureS1.jpg

- Figures2.jpg

- FigureS3.jpg

- FigureS4.png

- FigureS5.jpg

- Figures6.jpg

- FigureS7.jpg

- Supplementallnformation.docx 